\title{
Utilisation des procédés électrochimiques et leurs combinaisons avec les procédés biologiques pour le traitement des lixiviats de sites d'enfouissement sanitaires - revue de littérature \\ Use of electrochemical processes and their combinations with biological processes for sanitary landfill leachate treatment - Literature review
}

\author{
Oumar Dia, Patrick Drogui, Rino Dubé et Gerardo Buelna
}

Volume 29, numéro 1, 2016

Reçu le 6 janvier 2014, accepté le 22 octobre 2014

URI : https://id.erudit.org/iderudit/1035717ar

DOI : https://doi.org/10.7202/1035717ar

Aller au sommaire du numéro

Éditeur(s)

Université du Québec - INRS-Eau, Terre et Environnement (INRS-ETE)

ISSN

1718-8598 (numérique)

Découvrir la revue

Citer cet article

Dia, O., Drogui, P., Dubé, R. \& Buelna, G. (2016). Utilisation des procédés électrochimiques et leurs combinaisons avec les procédés biologiques pour le traitement des lixiviats de sites d'enfouissement sanitaires - revue de littérature. Revue des sciences de l'eau / Journal of Water Science, 29(1), 63-89. https://doi.org/10.7202/1035717ar
Résumé de l'article

Les lixiviats des sites d'enfouissement sanitaires contiennent divers types de composés organiques et inorganiques susceptibles de polluer les milieux aquatiques s'ils ne sont pas convenablement traités. Depuis quelques années, on note une utilisation croissante des techniques électrochimiques, avec des résultats satisfaisants, pour le traitement des lixiviats. Parmi les avantages liés à l'utilisation de ces procédés, on peut citer : une faible emprise au sol, un temps de traitement court, une utilisation limitée, voire inexistante, de réactifs chimiques et une facilité d'automatisation. La capacité à traiter les composés organiques bioréfractaires et à augmenter la biodégradabilité de l'effluent constitue un atout majeur lors du traitement de certains types de lixiviats. Dans cette revue de littérature, un accent particulier est porté sur les trois procédés électrochimiques les plus utilisés pour le traitement des lixiviats, à savoir : l'électro-oxydation (EO), l'électro-Fenton (EF) et l'électrocoagulation (EC). Pour chacun des procédés, les différents paramètres opératoires qui influencent l'efficacité du traitement sont élucidés. D'une façon globale, les procédés électrochimiques sont influencés entre autres par l’intensité du courant appliqué, le type d'électrode utilisé, la distance interélectrodes, le temps de traitement, le $\mathrm{pH}$ et la conductivité du milieu. Le couplage des procédés électrochimiques et biologiques pour le traitement des lixiviats a été investigué. L'intégration de ces deux procédés permet d'augmenter les performances épuratoires tout en réduisant les coûts et les temps de traitement. Toutefois, des études approfondies sont nécessaires afin d'optimiser ces couplages et d'éclaircir l'influence du traitement biologique sur le traitement électrochimique et vice-versa. 


\section{UTILISATION DES PROCÉDÉS ÉLECTROCHIMIQUES ET LEURS} COMBINAISONS AVEC LES PROCÉDÉS BIOLOGIQUES POUR

\section{LE TRAITEMENT DES LIXIVIATS DE SITES D'ENFOUISSEMENT SANITAIRES - REVUE DE LITTÉRATURE}

Use of electrochemical processes and their combinations with biological processes for sanitary landfill leachate treatment - Literature review

OUMAR DIA ${ }^{1}$, PATRICK DROGUI', RINO DUBÉ2 ET GERARDO BUELNA2

${ }^{1}$ Centre Eau Terre Environnement, Institut national de la recherche scientifique, 490, rue de la Couronne, Québec (Québec) G1K 9A9 Canada

${ }^{2}$ Centre de recherche industrielle du Québec, 333, rue Franquet, Québec (Québec) G1P 4C7 Canada

Reçu le 6 janvier 2014, accepté le 22 octobre 2014

\section{RÉSUMÉ}

Les lixiviats des sites d'enfouissement sanitaires contiennent divers types de composés organiques et inorganiques susceptibles de polluer les milieux aquatiques s'ils ne sont pas convenablement traités. Depuis quelques années, on note une utilisation croissante des techniques électrochimiques, avec des résultats satisfaisants, pour le traitement des lixiviats. Parmi les avantages liés à l'utilisation de ces procédés, on peut citer : une faible emprise au sol, un temps de traitement court, une utilisation limitée, voire inexistante, de réactifs chimiques et une facilité d'automatisation. La capacité à traiter les composés organiques bioréfractaires et à augmenter la biodégradabilité de l'effluent constitue un atout majeur lors du traitement de certains types de lixiviats. Dans cette revue de littérature, un accent particulier est porté sur les trois procédés électrochimiques les plus utilisés pour le traitement des lixiviats, à savoir : l'électro-oxydation (EO), l'électroFenton (EF) et l'électrocoagulation (EC). Pour chacun des procédés, les différents paramètres opératoires qui influencent l'efficacité du traitement sont élucidés. D'une façon globale, les procédés électrochimiques sont influencés entre autres par l'intensité du courant appliqué, le type d'électrode utilisé, la distance interélectrodes, le temps de traitement, le $\mathrm{pH}$ et la conductivité du milieu. Le couplage des procédés électrochimiques et biologiques pour le traitement des lixiviats a été investigué. L'intégration de ces deux procédés permet d'augmenter les performances épuratoires tout en réduisant les coûts et les temps de traitement. Toutefois, des études approfondies sont nécessaires afin d'optimiser ces couplages et d'éclaircir l'influence du traitement biologique sur le traitement électrochimique et vice-versa.

Mots-clés : Lixiviat, site d'enfouissement, traitement électrochimique, traitement biologique, électro-oxydation, électrocoagulation, oxydation anodique, électro-Fenton. 


\section{ABSTRACT}

Landfill leachates contain several types of organic and inorganic pollutants that can contaminate aquatic environments if they are not treated in an appropriate way. In recent years, there has been an increase in the use of electrochemical processes for the treatment of landfill leachates, with satisfactory results. Some advantages related to the use of electrochemical processes are: small space requirement, short treatment time, low requirements of chemical reagents and easy automation. The ability to remove biorefractory compounds and to increase the biodegradability of the effluent makes them interesting methods for the treatment of landfill leachates. In the present literature review, particular attention is paid to three electrochemical processes commonly used for the treatment of landfill leachates, namely Electro-Oxidation (EO), Electro-Fenton (EF) and Electro-Coagulation (EC). For each of them, we elucidate the different parameters that affect treatment efficiency. Generally, electrochemical processes are influenced by the applied current density, type of electrode, distance between electrodes, treatment time, $\mathrm{pH}$ and effluent conductivity. The combination of electrochemical processes with biological processes is also considered. The integration of these two processes improves the treatment efficiency for landfill leachates and reduces the cost and treatment time. However, further studies are required in order to optimize the combined method and to clarify the influence of biological treatment on the electrochemical treatment, and vice versa.

\section{Key Words: Landfill leachate, electrochemical treatment, biological treatment, anodic oxidation, electro-oxidation, electrocoagulation, electro-Fenton.}

\section{INTRODUCTION}

L'enfouissement est l'un des principaux modes de gestion des déchets dans plusieurs pays du monde (ORKUN et KULEYIN, 2012). Les déchets enfouis subissent divers processus de transformations chimiques et biologiques à l'intérieur de la décharge (KJELDSEN et al., 2002). La percolation de l'eau de pluie à travers la décharge entraîne la production d'un effluent chargé en matières organiques et minérales appelé lixiviat (LEMA et al., 1988). Vu l'impact négatif que ces lixiviats peuvent présenter pour l'environnement, une attention particulière doit être portée sur les sites d'enfouissement des déchets afin de préserver toute intrusion des lixiviats dans les nappes phréatiques (LEMA et al., 1988). La composition physico-chimique des lixiviats varie d'une décharge à une autre. La teneur en polluants dans les lixiviats est fortement influencée par l'âge de la décharge (KJELDSEN et al., 2002). Ainsi, les lixiviats issus des décharges " jeunes" sont caractérisés par une forte concentration en matières organiques principalement biodégradables (HARMSEN, 1983). Avec le vieillissement de la décharge, on note une diminution des concentrations du lixiviat et la présence de divers composés organiques réfractaires (EHRIG, 1989). Les procédés biologiques sont traditionnellement utilisés pour le traitement des lixiviats. Parmi les procédés biologiques couramment utilisés, on peut citer : le lagunage (ROBINSON et GRANTHAM, 1988), les boues activées (HOSOMI et al., 1989), les lits bactériens (MARTIENSSEN et al., 1995), etc. Les procédés biologiques présentent comme principal atout l'efficacité de traiter les polluants organiques biodégradables avec un coût rentable (KURNIAWAN et al., 2010). Ils sont aussi efficaces pour le traitement des lixiviats issus des décharges " jeunes " caractérisés par leur biodégradabilité. Cependant, leur utilisation est limitée pour l'épuration des lixiviats composés de grands pourcentages de composés bioréfractaires (XIAO et al., 2013). Pour faire face à ce problème, quelques procédés physico-chimiques ont été expérimentés au cours de ces dernières années pour le traitement des lixiviats de sites d'enfouissement sanitaires. Les procédés membranaires, les procédés d'oxydation avancée et les procédés électrochimiques occupent une place importante dans ces nouvelles technologies de traitement. Les membranes filtrantes (microfiltration, ultrafiltration, nanofiltration et osmose inverse) permettent de transférer les polluants d'une phase à une autre sans pour autant les dégrader. De plus, les phénomènes de colmatage des membranes rendent leur utilisation délicate (RENOU et al., 2008). Les procédés d'oxydation avancée permettent une dégradation partielle ou totale des polluants avec l'utilisation d'oxydants puissants comme l'ozone et le radical hydroxyle combinés avec les radiations ultraviolettes et les ultrasons. Malgré les bonnes efficacités des procédés d'oxydation avancée, ils nécessitent une grande consommation énergétique liée à l'utilisation des générateurs d'ozone, des lampes UV et des générateurs d'ultrasons (LOPEZ et al., 2004). Certains de ces procédés requièrent également une utilisation conséquente de peroxyde d'hydrogène et de sulfate ferreux (procédé Fenton). Quant aux procédés électrochimiques, ils permettent de générer de façon électrochimique des agents réactifs capables d'éliminer efficacement les polluants. Les avantages de tels procédés résident dans leurs aspects non polluants, leurs facilités d'automatisation, des temps de traitement réduits et des besoins en réactifs faibles (DROGUI et al., 2007). L'électro-oxydation (EO), l'électro-Fenton (EF) et l'électrocoagulation (EC) sont les trois procédés électrochimiques les plus couramment utilisés pour le traitement des lixiviats.

L'objectif de cette revue de littérature est double: a) identifier les différents paramètres opératoires qui influencent l'efficacité de traitement des technologies électrochimiques, b) étudier la faisabilité et les performances du couplage des procédés électrochimiques et biologiques pour le traitement des lixiviats de sites d'enfouissement sanitaires. 


\section{CARACTÉRISTIQUES DES LIXIVIATS} DE SITES D'ENFOUISSEMENT

Le débit et la composition des lixiviats sont influencés par différents paramètres tels que : la nature des déchets enterrés, l'âge de la décharge, le climat local (température, humidité, précipitation), le caractère géologique du site, le mode d'exploitation de la décharge (hauteur des déchets, degré de compacité), etc. (VADILLO et al., 1999; XIE et al., 2010a).

On distingue quatre étapes de décomposition des déchets dans la décharge au cours du temps : 1) la phase initiale aérobie; 2) la phase acidogène anaérobie; 3) la phase méthanogène initiale; 4) la phase méthanogène stable (KJELDSEN et al., 2002). Même après la phase de maturation stable d'une décharge, cette dernière continue à produire des lixiviats. Aux États-Unis, les décharges sont sur surveillance jusqu'à 30 ans suivant leur fermeture. On estime qu'après cette période, la décharge est complètement stable et qu'une surveillance intensive n'est plus nécessaire (KJELDSEN et al., 2002).

D'après CHRISTENSEN et al. (1994), la composition des lixiviats peut être divisée en quatre groupes : la matière organique dissoute, les macro-composés inorganiques, les métaux lourds et les composés organiques xénobiotiques. Il est à noter que les concentrations de ces différents polluants peuvent varier fortement d'un site à un autre et même au sein d'une même décharge. De ce fait, il est difficile de définir une composition typique et universelle des lixiviats. Dans la littérature, les concentrations sont souvent données par intervalles de valeurs.

De nombreuses études ont mis en évidence la diminution de la concentration en polluants organiques (demande chimique en oxygène [DCO] et demande biochimique en oxygène $[\mathrm{DBO}]$ ) avec l'augmentation de l'âge de la décharge (Tableau 1). Les lixiviats dits "jeunes" sont caractérisés par de fortes concentrations en polluants organiques avec un grand pourcentage de matières biodégradables composées essentiellement d'acide gras volatil (HARMSEN, 1983). La teneur élevée en matière organique est due à la fermentation anaérobique des composés organiques lors de la phase acidogène. Au cours des processus de fermentation et de méthanisation, une grande partie de la pollution organique est dégradée par voie biologique par la flore microbienne présente dans la décharge (KJELDSEN et al., 2002). Avec le temps, la décharge se stabilise, produisant ainsi un lixiviat « mature » ou "stable " caractérisé par une pollution organique qui présente une partie importante de composés réfractaires difficilement biodégradables (EHRIG, 1989). Le rapport de biodégradabilité (DBO/DCO) est généralement inférieur à 0,1 dans les lixiviats stables. Les substances humiques (acides humiques et fulviques) représentent la majeure partie de la pollution réfractaire des lixiviats (BARLAZ et HAM, 1993). D'après une étude de CHRISTENSEN et al. (1998) réalisée sur un lixiviat en phase méthanogène, le carbone organique dissous (COD) est composé de $60 \%$ d'acide fulvique, $30 \%$ de composés hydrophiles et $10 \%$ d'acide humique. Les poids moléculaires moyens des acides fulviques, des composés hydrophiles et des acides humiques sont respectivement de 1800,2100 et $2600 \mathrm{Da}$ (CHRISTENSEN et al., 1998). Entre les phases de méthanisation et de stabilisation, le lixiviat produit est dit "intermédiaire ». Il est caractérisé par un rapport de biodégradabilité compris entre 0,1 et 0,5 avec des concentrations en DCO généralement inférieures à $10000 \mathrm{mg} \cdot \mathrm{L}^{-1}$.

L'humidité joue un rôle important sur la vitesse de décomposition des déchets (WREFORD et al., 2000). Il s'avère que les déchets enterrés dans les régions arides se décomposent moins lentement par rapport aux déchets enfouis dans un sol qui reçoit beaucoup de précipitations (KJELDSEN et al., 2002).

Les lixiviats présentent généralement une bonne conductivité électrique liée à une forte concentration en ions $\mathrm{Cl}^{-}$et $\mathrm{NH}_{4}^{+}$(VADILLO et al., 1999). Le pH acide obtenu lors de la phase acidogène donne un caractère agressif aux lixiviats et favorise ainsi la dissolution des composés minéraux (KJELDSEN et al., 2002). Contrairement à la matière organique, la concentration en azote ammoniacal ne baisse pas de manière significative au cours du temps (BURTON et WATSON-CRAIK, 1998). Même dans les lixiviats «matures", on retrouve des concentrations élevées en azote ammoniacal (AMOKRANE et al., 1997; SINGH et al., 2012). Cela est dû en partie à l'ammonification de substances azotées comme les protéines et les acides aminés (BURTON et WATSONCRAIK, 1998) et le milieu anaérobie de la décharge qui ne favorise pas les réactions de nitrification. De ce fait, la pollution azotée est considérée comme un polluant à long terme dans les décharges (KJELDSEN et al., 2002).

Le tableau 2 présente les étendues de concentrations en métaux toxiques $(\mathrm{Cd}, \mathrm{Cr}, \mathrm{Cu}, \mathrm{Pb}, \mathrm{Ni}, \mathrm{Zn})$ pouvant être décelées dans les lixiviats. Ces concentrations sont faibles et sont pour la plupart inférieures aux normes en vigueur portant sur la qualité de l'eau potable (RULE, 1979; EHRIG, 1983; KJELDSEN et al., 2002; OYGARD et al., 2004). Sur 20 décharges étudiées, les concentrations en métaux étaient pour la plupart inférieures à $1 \mathrm{mg} \cdot \mathrm{L}^{-1}$ (EHRIG, 1983). Ces faibles concentrations sont dues à une très faible proportion $(0,02 \%)$ de métaux lixiviés par rapport à la teneur initiale introduite dans la décharge (KJELDSEN et al., 2002). Ces faibles concentrations sont également attribuées à l'adsorption des métaux à la surface des colloïdes et à la co-précipitation des métaux avec certains composés organiques et inorganiques présents dans les lixiviats (JENSEN et al., 1999; CALACE et al., 2001; BAUN et 
Tableau 1. Caractéristiques de différents lixiviats en fonction de l'âge de la décharge.

Table 1. Landfill leachate characteristics as a function of landfill age.

\begin{tabular}{|c|c|c|c|c|c|c|c|c|}
\hline $\begin{array}{l}\text { Âge de la } \\
\text { décharge }\end{array}$ & Pays & $\mathrm{pH}$ & $\begin{array}{c}\text { Conductivité } \\
\left(\mathbf{m S} \cdot \mathbf{c m}^{-1}\right)\end{array}$ & $\begin{array}{c}\mathrm{DCO}^{\mathrm{a}} \\
\left(\mathbf{m g} \cdot \mathrm{L}^{-1}\right)\end{array}$ & $\begin{array}{c}\mathrm{DBO}^{\mathrm{b}} \\
\left(\mathbf{m g} \cdot \mathrm{L}^{-1}\right)\end{array}$ & DBO/DCO & $\begin{array}{l}\mathrm{N}-\mathrm{NH}_{4} \\
\left(\mathbf{m g} \cdot \mathrm{L}^{-1}\right)\end{array}$ & Références \\
\hline Jeune & Canada & $5,7-6,4$ & - & 15000 & 13500 & 0,9 & 350 & HENRY et PRASAD, 2000 \\
\hline Jeune & Turquie & 7,95 & - & 22255 & 14900 & 0,67 & 2410 & KABDAŞLI et al., 2008 \\
\hline Jeune & Corée du Sud & $7,2-7,6$ & - & $21300-26940$ & $9250-11650$ & 0,43 & $1635-1810$ & IM et al., 2001 \\
\hline Jeune & Grèce & $4,9-6,7$ & $23-35,5$ & $44000-115000$ & $9500-80800$ & $0,21-0,7$ & $1400-10250$ & TATSI et al., 2003) \\
\hline Intermédiaire & Canada & $7,7-7,9$ & - & 1500 & 450 & 0,3 & 600 & HENRY et PRASAD, 2000 \\
\hline Intermédiaire & Portugal & 8,5 & 36,1 & 21700 & 6500 & 0,3 & 1200 & FERNANDES et al., 2014 \\
\hline Intermédiaire & Chine & $6,4-7,3$ & & 2566 & 960 & 0,37 & 386 & LI et al., 2011 \\
\hline Intermédiaire & Italie & 8,4 & 20,5 & 5050 & 1270 & 0,25 & 1330 & FRASCARI et al., 2004 \\
\hline Mature & France & 8,2 & - & 4100 & 200 & 0,05 & 1040 & AMOKRANE et al., 1997 \\
\hline Mature & Japon & 8,1 & - & $86-221$ & 26 & $0,01-0,12$ & $104-332$ & IMAI et al., 1995 \\
\hline Mature & États-Unis & 7,7 & 13,1 & 2300 & 46 & 0,02 & 1060 & SINGH et al., 2012 \\
\hline Mature & Finlande & - & $3,07-7,08$ & $270-1000$ & $25-170$ & $0,09-0,17$ & $53-270$ & HOILIJOKI et al., 2000 \\
\hline
\end{tabular}

${ }^{a} \mathrm{DCO}:$ demande chimique en oxygène

${ }^{b} \mathrm{DBO}:$ demande biochimique en oxygène

Tableau 2. Concentrations des métaux lourds dans les lixiviats (KJELDSEN et al., 2002).

Table 2. Heavy metal concentrations in landfill leachates.

\begin{tabular}{lc}
\hline $\begin{array}{l}\text { Métaux } \\
\text { lourds }\end{array}$ & $\begin{array}{c}\text { Étendue des concentrations } \\
\left(\mathbf{m g} \cdot \mathbf{L}^{-1}\right)\end{array}$ \\
\hline Arsenic & $1 \cdot 10^{-2}-1$ \\
Cadmium & $1 \cdot 10^{-4}-0,4$ \\
Chrome & $2 \cdot 10^{-2}-1,5$ \\
Cobalt & $5 \cdot 10^{-3}-1,5$ \\
Cuivre & $5 \cdot 10^{-3}-10$ \\
Plomb & $1 \cdot 10^{-3}-5$ \\
Mercure & $1,5 \cdot 10^{-5}-0,16$ \\
Nickel & $1,5 \cdot 10^{-2}-13$ \\
Zinc & $3 \cdot 10^{-2}-1 \cdot 10^{3}$ \\
\hline
\end{tabular}

CHRISTENSEN, 2004). Ces métaux peuvent, par exemple, précipiter sous forme de sulfures et de carbonates. Ces composés sont généralement présents en quantités non négligeables dans les lixiviats (KJELDSEN et al., 2002).

De nombreuses études ont démontré la toxicité des lixiviats issus des sites d'enfouissement sanitaires (PLOTKIN et RAM, 1984; CLÉMENT et MERLIN, 1995; OSHODE et al., 2008). CHEUNG et al. (1993) et CLÉMENT et MERLIN (1995) ont rapporté que la concentration élevée en azote ammoniacal contribue de façon considérable à la toxicité des lixiviats. La présence des microorganismes pathogènes contribue aussi à la toxicité des lixiviats (OSHODE et al., 2008). Lors d'une étude de la population bactérienne d'un lixiviat, OSHODE et al. (2008) ont recensé 112 espèces bactériennes appartenant à 17 genres différents. Certains microorganismes pathogènes recensés (Bacillus cereus, Escherichia coli, Staphylococus aureus, Clostridium sordelli, etc.) produisent des substances toxiques dans le milieu. D'autres facteurs comme le $\mathrm{pH}$, la conductivité et la présence de certains métaux lourds peuvent contribuer à la toxicité des lixiviats (KJELDSEN et al., 2002).

Les lixiviats contiennent une large variété de composés xénobiotiques dont les plus fréquemment rencontrés sont les hydrocarbures aromatiques (benzène, toluène, éthylbenzène, xylènes, etc.) et les hydrocarbures halogénés comme le tétrachloroéthylène et le trichloréthylène (KJELDSEN et al., 2002).

\section{PROCÉDÉ D'ÉLECTRO-OXYDATION (EO)}

Le procédé d'oxydation anodique est utilisé depuis quelques décennies pour le traitement de la couleur et de certains polluants organiques tels que le phénol, les cyanures et l'aniline (LANZA et BERTAZZOLI, 2002; CANIZARES et al., 2005b). Ces dernières années, son utilisation pour le traitement des lixiviats a donné des résultats relativement satisfaisants. Il est souvent utilisé comme étape de prétraitement pour réduire la charge de la DCO et de l'azote ammoniacal ou encore comme traitement tertiaire pour la dégradation des polluants organiques récalcitrants (FERNANDES et al., 2012). On distingue deux types de réaction : la réaction d'oxydation directe et la réaction d'oxydation indirecte (Figure 1).

Dans une réaction d'oxydation directe, l'oxydation se fait soit par conversion électrochimique ou par combustion électrochimique (COMNINELLIS, 1994). Pendant la conversion électrochimique, les composés organiques non biodégradables sont partiellement oxydés en composés plus biodégradablestandisquelors dela combustion électrochimique, 
a)

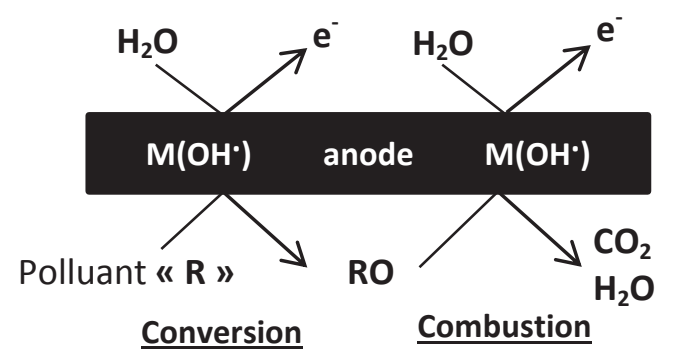

b)

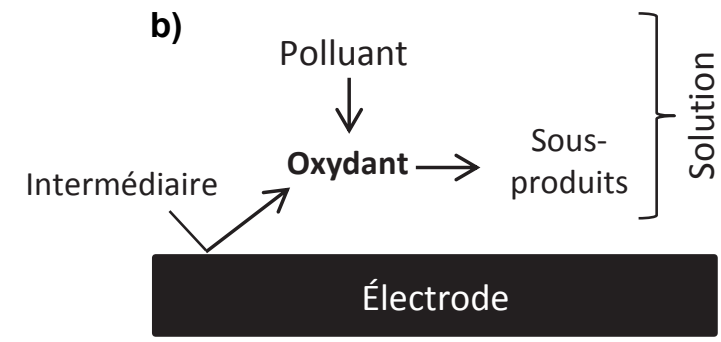

Figure 1. Processus d'oxydation directe (a) et indirecte (b).

Mechanisms of direct (a) and indirect oxidation (b).

on assiste à une dégradation complète des polluants organiques sous forme de $\mathrm{CO}_{2}$ et de $\mathrm{H}_{2} \mathrm{O}$ (GRIMM et al., 1998). La réaction directe se fait en deux étapes : une première étape d'oxydation anodique de la molécule d'eau conduisant à la formation de l'espèce réactive $\mathrm{OH}^{*}$ qui s'adsorbe sur un site actif de l'électrode $\mathrm{M}\left(\mathrm{OH}^{\circ}\right)$ (Réaction 1) et une seconde étape où le radical hydroxyle oxyde le polluant organique $\mathrm{R}$ en $\mathrm{RO}$ (Réaction 2). La réaction entre les composés organiques oxydés $\mathrm{RO}$ et les radicaux hydroxyles peut conduire à une oxydation complète (DROGUI et al., 2007) (Équation 3).

$$
\begin{gathered}
\mathrm{H}_{2} \mathrm{O}+\mathrm{M} \rightarrow \mathrm{M}\left(\mathrm{OH}^{\bullet}\right)+\mathrm{H}^{+}+\mathrm{e}^{-} \\
\mathrm{R}+\mathrm{M}\left(\mathrm{OH}^{\bullet}\right) \rightarrow \mathrm{M}+\mathrm{RO}+\mathrm{e}^{-} \\
\mathrm{R}+\mathrm{M}\left(\mathrm{OH}^{\bullet}\right) \rightarrow \mathrm{M}+m \mathrm{CO}_{2}+n \mathrm{H}_{2} \mathrm{O}+\mathrm{H}^{+}+\mathrm{e}^{-}
\end{gathered}
$$

Un phénomène de compétition peut avoir lieu pendant l'oxydation et diminuer ainsi l'efficacité de dégradation des polluants (DROGUI et al., 2007). La formation d'oxygène est un exemple de réaction parasite (Équation 4).

$$
\mathrm{H}_{2} \mathrm{O}+\mathrm{M}\left(\mathrm{OH}^{\bullet}\right) \rightarrow \mathrm{M}+\mathrm{O}_{2}+3 \mathrm{H}^{+}+3 \mathrm{e}^{-}
$$

Pendant l'oxydation indirecte, les substances organiques sont détruites par des intermédiaires réactifs tels que le peroxyde d'hydrogène $\left(\mathrm{H}_{2} \mathrm{O}_{2}\right)$, l'ozone $\left(\mathrm{O}_{3}\right)$, l'acide peroxydisulfurique $\left(\mathrm{H}_{2} \mathrm{~S}_{2} \mathrm{O}_{8}\right)$, l'acide hypochloreux $(\mathrm{HClO})$, l'acide hypobromeux ( $\mathrm{HBrO})$ ou d'autres oxydants formés par électro-oxydation des composés inorganiques présents dans la solution (GRIMM et al., 1998; DROGUI et al., 2001; STUCKI et al., 1987; DENG et ENGLEHARDT, 2007; FARMER et al., 1992) (équations 5 à 7 ).

$$
2 \mathrm{SO}_{4}^{2-}+2 \mathrm{H}^{+} \rightarrow \mathrm{H}_{2} \mathrm{~S}_{2} \mathrm{O}_{8}+2 \mathrm{e}^{-}
$$

$$
\begin{gathered}
\mathrm{Cl}^{-}+2 \mathrm{H}_{2} \mathrm{O} \rightarrow \mathrm{HClO}+\mathrm{H}_{3} \mathrm{O}^{+}+2 \mathrm{e}^{-} \\
\mathrm{O}_{2}+2 \mathrm{H}^{+}+2 \mathrm{e}^{-} \rightarrow \mathrm{H}_{2} \mathrm{O}_{2}
\end{gathered}
$$

$\mathrm{Vu}$ que l'objectif du traitement électrochimique est de dégrader les polluants organiques, il est préférable d'utiliser des électrodes qui ont une forte surtension d'oxygène (DROGUI et al., 2007). Les anodes les plus utilisées sont : les oxydes de titane et de ruthénium recouverts de titane (DSA), les électrodes ternaires $\mathrm{Sn}-\mathrm{Pb}-\mathrm{Ru}$ recouvertes de titane (SPR), le diamant dopé au bore (DDB), le graphite, etc. (FEKI et al., 2009). L'usage de ces électrodes à forte surtension d'oxygène implique la génération d'espèces oxygénées réactives, comme le radical hydroxyle, responsables de la dégradation de la matière organique (WEISS, 2006). Ces matériaux présentent également une stabilité chimique par rapport aux milieux acides et alcalins. La cathode est souvent constituée par un élément métallique simple comme l'acier inoxydable, le cuivre ou le titane (CHIANG et al., 1995; MORAES et BERTAZZOLI, 2005; TSAI et al., 1997). Le choix de l'électrode peut influencer de manière significative les performances du traitement. En utilisant différents types d'électrodes à l'anode, CHIANG et al. (1995) ont obtenu des taux décroissants d'abattement de la DCO selon cet ordre SPR > DSA (oxyde de Ru et de Ti recouvert de $\mathrm{Ti}$ i) $>\mathrm{PbO}_{2} / \mathrm{Ti}>$ graphite. Un taux d'abattement de $92 \%$ de la DCO et une élimination quasi complète de l'azote ammoniacal sont obtenus avec l'anode en SPR et une densité de courant de $150 \mathrm{~mA} \cdot \mathrm{cm}^{-2}$. Les taux d'abattement satisfaisants obtenus avec cette électrode (SPR) sont liés à la production d'oxydants intermédiaires, tels que $\mathrm{HClO}$, formés lors de l'oxydation indirecte. Selon les auteurs, le SPR présente plus de capacité à générer les oxydants intermédiaires par rapport aux autres électrodes étudiées. Pour combiner à la fois les effets d'électro-oxydation et d'électrocoagulation, TSAI et al. (1997) ont utilisé le fer et l'aluminium comme anodes solubles; ils ont obtenu un pourcentage d'élimination de la DCO variant de 30 à $50 \%$. L'électrode de diamant dopé au bore (DDB) a 
été utilisée par CABEZA et al. (2007) pour le traitement de l'azote ammoniacal avec des performances dépassant $95 \%$ d'abattement.

La concentration en ions chlorures $\left(\mathrm{Cl}^{-}\right)$et la densité de courant appliquée jouent également un rôle important dans l'efficacité du traitement des lixiviats (DENG et ENGLEHARDT, 2007). Les lixiviats de décharge peuvent contenir des concentrations en ions chlorures variant entre 150 et $4500 \mathrm{mg} \cdot \mathrm{L}^{-1}$ (KJELDSEN et al., 2002). L'oxydation anodique des ions chlorures en chlore gazeux est suivie de sa dismutation en solution et conduit à la formation d'acide hypochloreux $(\mathrm{HClO})$. Cependant, le chlore peut réagir avec des composés organiques et former des composés organochlorés dont certains sont potentiellement cancérigènes (DENG et ENGLEHARDT, 2007). Selon LI et al. (2001), la concentration en ions chlorures a un effet positif sur l'abattement de la DCO jusqu'à une concentration de $5000 \mathrm{mg} \cdot \mathrm{L}^{-1}$. Au-delà de cette valeur, l'ajout supplémentaire de chlorures n'améliore pas la performance du procédé. En présence d'ion sulfates, ceux-ci peuvent également être oxydés en acide persulfurique $\left(\mathrm{H}_{2} \mathrm{~S}_{2} \mathrm{O}_{8}\right)$ sur une anode constituée de diamant dopé au bore (DDB) (SERRANO et al., 2002). L'acide persulfurique est un oxydant puissant pouvant être utilisé pour l'oxydation de la matière organique dans les lixiviats. En somme, les composés organiques peuvent être directement oxydés à l'anode et indirectement oxydés en solution par l'acide hypochloreux ou par l'acide persulfurique.

Comme tous les procédés électrochimiques, l'intensité appliquée est un facteur majeur de l'opération. L'étendue des densités de courant utilisés dans le traitement des lixiviats varie d'un minimum de $5 \mathrm{~mA} \cdot \mathrm{cm}^{-2}$ à un maximum de $540 \mathrm{~mA} \cdot \mathrm{cm}^{-2}$ (DENG et ENGLEHARDT, 2007). Laugmentation de la densité de courant augmente les performances de dépollution en termes de DCO, d'azote ammoniacal et de couleur (MORAES et BERTAZZOLI, 2005; CHIANG et al., 1995). D'après une étude de CHIANG et al. (1995), en augmentant la densité de courant de 50 à $150 \mathrm{~mA} \cdot \mathrm{cm}^{-2}$, le taux d'élimination de l'azote ammoniacal a doublé de 40 à $80 \%$ tandis que l'abattement de la DCO a augmenté de 25 à $35 \%$. Cependant, l'augmentation du courant peut engendrer la coloration de la solution suite à la corrosion de l'anode. BASHIR et al. (2009) ont observé une coloration du lixiviat et une chute de l'abattement de la DCO due à la corrosion de l'anode en graphite utilisée. Un phénomène similaire est observé par RADA et al. (2013) suite à la corrosion de l'électrode en acier. Ces effets néfastes liés à la corrosion des électrodes montrent l'importance de choisir des électrodes qui présentent à la fois une forte surtension en oxygène et une bonne stabilité chimique. Il est à noter que travailler avec des densités de courant faibles peut être néfaste pour l'opération d'EO. COSSU et al. (1998) ont observé une coloration de la solution et un dépôt de précipité marron sur la surface des anodes de $\mathrm{Ti} / \mathrm{PbO}_{2}$ et $\mathrm{Ti} / \mathrm{SnO}_{2}$ en travaillant avec une densité de courant inférieure à $5 \mathrm{~mA} \cdot \mathrm{cm}^{-2}$.
Le temps de traitement a aussi une influence sur l'efficacité du traitement. Son augmentation favorise généralement la dégradation des polluants. En augmentant le temps de traitement de 0,5 à 4 h, CHIANG et al. (1995) ont observé une augmentation de 5 à $30 \%$ du taux d'élimination de la DCO et d'environ $20 \%$ pour l'azote ammoniacal. L'influence du $\mathrm{pH}$ sur le traitement reste un sujet controversé (DENG et ENGLEHARDT, 2007). D'après COSSU et al. (1998), seule une légère augmentation de la vitesse de dégradation de la DCO est obtenue après avoir fait varier le $\mathrm{pH}$ du lixiviat de 8,3 à 3,0. Cependant, LI et al. (2001) et VLYSSIDES et al. (2003) suggèrent des $\mathrm{pH}$ acides pour l'optimisation de la consommation électrique et l'abattement de la DCO. La modeste hausse de l'abattement de la DCO en milieu acide est attribuée à la faible concentration en ions carbonates et bicarbonates retrouvés pour de faibles valeurs de $\mathrm{pH}$. Ces espèces alcalines peuvent entrer rapidement en réaction avec les radicaux hydroxyles produits et limiter ainsi l'efficacité de la dégradation des composés organiques (COSSU et al., 1998). Le tableau 3 résume les résultats de quelques études sur l'électro-oxydation des lixiviats.

\section{PROCÉDÉ D’ÉLECTRO-FENTON (EF)}

L'action du réactif de Fenton sur l'acide tartrique a été observée par Fenton (FENTON, 1894). Depuis lors, cette technique est utilisée pour dégrader différents composés organiques présents dans les eaux usées. Dans le procédé traditionnel de Fenton, le peroxyde d'hydrogène $\mathrm{H}_{2} \mathrm{O}_{2}$ réagit avec l'ion ferreux $\mathrm{Fe}^{2+}$ pour former le radical hydroxyle $\mathrm{OH}^{\circ}$ qui est un oxydant puissant non sélectif $(\mathrm{E} \approx 2,73 \mathrm{~V})$ (YAMAZAKI et PIETTE, 1991) (Équation 8). Ce dernier est capable de dégrader la majeure partie des composés organiques jusqu’à l'étape ultime de la minéralisation.

$$
\begin{gathered}
\mathrm{H}_{2} \mathrm{O}_{2}+\mathrm{Fe}^{2+} \rightarrow \mathrm{Fe}^{3+}+\mathrm{OH}^{\bullet}+\mathrm{OH}^{-} \\
k_{8}=70 \mathrm{M}^{-1} \cdot \mathrm{s}^{-1}
\end{gathered}
$$

En dehors de la réaction principale de Fenton, une série de réactions secondaires aura lieu dans le milieu réactionnel (équations 9 à 14) (UMAR et al., 2010).

$$
\begin{gathered}
\mathrm{Fe}^{3+}+\mathrm{H}_{2} \mathrm{O}_{2} \rightarrow \mathrm{Fe}^{2+}+\mathrm{HO}_{2}^{\bullet}+\mathrm{H}^{+} \\
k_{9}=10^{-3}-10^{-2} \mathrm{M}^{-1} \cdot \mathrm{s}^{-1} \\
\mathrm{OH}^{\bullet}+\mathrm{H}_{2} \mathrm{O}_{2} \rightarrow \mathrm{HO}_{2}{ }_{2}+\mathrm{H}_{2} \mathrm{O} \\
k_{10}=3,3 \cdot 10^{7} \mathrm{M}^{-1} \cdot \mathrm{s}^{-1}
\end{gathered}
$$




$$
\begin{gathered}
\mathrm{OH}^{\bullet}+\mathrm{Fe}^{2+} \rightarrow \mathrm{Fe}^{2+}+\mathrm{OH}^{-} \\
k_{11}=3 \cdot 10^{8} \mathrm{M}^{-1} \cdot \mathrm{s}^{-1} \\
\mathrm{Fe}^{3+}+\mathrm{HO}_{2}^{\bullet} \rightarrow \mathrm{Fe}^{2+}+\mathrm{O}_{2} \mathrm{H}^{+} \\
\mathrm{Fe}^{2+}+\mathrm{HO}_{2}{ }_{2} \rightarrow \mathrm{Fe}^{3+}+\mathrm{H}_{2} \mathrm{O}_{2} \\
2 \mathrm{HO}_{2} \rightarrow \mathrm{H}_{2} \mathrm{O}_{2}+\mathrm{O}_{2}
\end{gathered}
$$

Même si la vitesse de formation du radical hydroxyle dans la réaction 8 est assez rapide $\left(k_{8}=70 \mathrm{M}^{-1} \cdot \mathrm{s}^{-1}\right)$, l'ion ferreux et le peroxyde d'hydrogène entrent très rapidement en réaction avec le radical $\mathrm{OH}^{*}$ à une cinétique très rapide : $k_{10}=3,3 \cdot 10^{7} \mathrm{M}^{-1} \cdot \mathrm{s}^{-1}$ pour $\mathrm{H}_{2} \mathrm{O}_{2}$ et $k_{11}=3,2 \cdot 10^{8} \mathrm{M}^{-1} \cdot \mathrm{s}^{-1}$ pour $\mathrm{Fe}^{2+}$ (équations 10 et 11) (BUXTON et al., 1988). De plus, la réaction de la régénération du $\mathrm{Fe}(\mathrm{II})$ à partir du $\mathrm{Fe}(\mathrm{III})$ par le peroxyde d'hydrogène (Réaction 9) se fait avec une cinétique lente $\left(k_{9}=10^{-3}-10^{-2} \mathrm{M}^{-1} \cdot \mathrm{s}^{-1}\right)$ (WALLING et GOOSEN, 1973). Ces phénomènes peuvent limiter la disponibilité du radical hydroxyle dans la solution et diminuer par conséquent l'efficacité de dégradation des polluants. En outre, les quantités importantes de fer(II) et de peroxyde ajoutées pour maintenir la productivité du radical hydroxyle peuvent entraîner un surplus de production des boues d'hydroxydes ferrique suite à la coagulation des colloïdes par le fer(III) (CHOU et al., 1999).

Pour augmenter l'efficacité du procédé conventionnel de Fenton, une nouvelle technique mettant en synergie le procédé d'électro-oxydation et la réaction de Fenton est mise au point : l'électro-Fenton $(\mathrm{EF})$. Cette méthode présente comme principaux avantages : la génération in situ du $\mathrm{Fe}(\mathrm{II})$, la régénération électrochimique du fer(II) à partir du fer(III) et la formation in situ du peroxyde d'hydrogène à partir de la réduction de l'oxygène dissous (Équation 15) (ZHANG et al., 2006; QIANG et al., 2003).

$$
\mathrm{O}_{2}+2 \mathrm{H}^{+}+2 \mathrm{e}^{-} \rightarrow \mathrm{H}_{2} \mathrm{O}_{2}
$$

Selon les besoins du traitement, l'opération d'électroFenton peut être réalisée selon quatre scénarios (QIANG et al., 2003) :

1. Ajout du $\mathrm{Fe}(\mathrm{II})$ et du peroxyde et régénération du $\mathrm{Fe}(\mathrm{II})$ par réduction cathodique du $\mathrm{Fe}(\mathrm{III})$;

2. Ajout du peroxyde et formation anodique de Fe(II) suivie de la régénération du $\mathrm{Fe}(\mathrm{II})$ par réduction cathodique du $\mathrm{Fe}(\mathrm{III})$;

3. Ajout du Fe(II), formation cathodique du peroxyde à partir de l'oxygène dissous et régénération du Fe(II) par réduction cathodique du $\mathrm{Fe}(\mathrm{III})$;
4. Formation cathodique du peroxyde à partir de l'oxygène dissous et formation anodique du $\mathrm{Fe}(\mathrm{II})$ suivie de la régénération du $\mathrm{Fe}(\mathrm{II})$ par réduction cathodique du Fe(III).

Les électrodes utilisées pour la réalisation de l'électroFenton different selon les besoins du procédé. Ainsi, le fer et ses alliages sont largement utilisés comme anodes sacrificielles pour la génération électrochimique des ions ferreux (LIN et CHANG, 2000; BRILLAS et CASADO, 2002; ATMACA, 2009). Différents types de cathodes tels que l'acier inoxydable, le titane, le graphite, le feutre de carbone, le carbone PTFE (polytétrafluoroéthylène), etc. sont utilisés pour réduire le fer(III) en fer(II) (QIANG et al., 2003; BRILLAS et CASADO, 2002; ZHANG et al., 2012a; CHOU et al., 1999). Pour minimiser l'oxydation du fer(II) en fer(III) à l'anode, CHOU et al. (1999) préconisent un ratio de surface anode/cathode de 3:8. Concernant la génération cathodique du peroxyde d'hydrogène, les électrodes les plus couramment rencontrées sont le carbone vitreux réticulé et le carbone PTFE (CHOU et al., 1999).

Le procédé d'électro-Fenton est utilisé depuis quelques années pour traiter divers polluants organiques. Cependant, son application pour le traitement des lixiviats n'est pas encore très répandue comparée à l'utilisation du procédé de Fenton classique (UMAR et al., 2010). Les études existantes effectuées sur les lixiviats montrent des résultats satisfaisants en termes d'abattement de la DCO, de la couleur et des germes pathogènes (MOHAJERI et al., 2010; TRABELSI et al., 2012; ZHANG et al., 2012a; YOON et al., 1998; AZIZ et al., 2013). Le tableau 4 résume les résultats obtenus de quelques études d'électro-Fenton réalisées sur des lixiviats issus de sites d'enfouissement.

Les principaux facteurs qui influencent l'efficacité du traitement sont : le $\mathrm{pH}$, l'intensité du courant, les doses de réactifs utilisées, le ratio molaire $\mathrm{H}_{2} \mathrm{O}_{2} / \mathrm{Fe}^{2+}$ et la distance interélectrodes. Le $\mathrm{pH}$ est l'un des facteurs les plus influents sur l'efficacité du procédé de Fenton. Les $\mathrm{pH}$ faibles sont favorables à la formation du peroxyde d'hydrogène, car la réduction de l'oxygène en peroxyde se fait en milieu acide (Équation 15) (WANG et al., 2010). L'étendue optimale de $\mathrm{pH}$ est très restreinte, elle se situe entre 2 et 4,5 (DENG et ENGLEHARDT, 2006). Des valeurs de $\mathrm{pH}$ supérieures ou inférieures à cette plage optimale entraînent des répercussions sur la qualité du traitement. À des $\mathrm{pH}$ très acides $(\mathrm{pH}<2)$, le piégeage des radicaux hydroxyles par les protons $\mathrm{H}^{+}$est accéléré (TANG et HUANG, 1996) et les ions ferreux forment des complexes stables avec le peroxyde d'hydrogène limitant ainsi la réaction de Fenton (NIDHEESH et GANDHIMATHI, 2012). Si le $\mathrm{pH}$ est supérieur au $\mathrm{pH}$ optimal $(\mathrm{pH}>4,5)$, la régénération du fer(II) à partir du Fe(III) est limitée par le phénomène de coagulation des colloïdes par les ions fer(II) et fer(III) (MOLLAH et al., 2001). Les autres effets défavorables liés à 
Tableau 3. Résumé des résultats de quelques études sur l'électro-oxydation des lixiviats de sites d'enfouissement.

Table 3. Summary of results of some studies on electro-oxidation of landfill leachates.

\begin{tabular}{|c|c|c|c|c|c|c|c|c|c|c|}
\hline Anode & Cathode & $\begin{array}{l}\text { Volume } \\
\text { de travail } \\
\text { (L) }\end{array}$ & $\underset{\text { (initial) }}{\mathrm{pH}}$ & $\begin{array}{l}\text { Durée de } \\
\text { traitement } \\
(\text { min) }\end{array}$ & $\begin{array}{c}\text { Concentration } \\
\text { DCO }^{\mathrm{c}} \text { initiale } \\
\left(\mathrm{mg} \cdot \mathrm{L}^{-1}\right)\end{array}$ & $\begin{array}{c}\text { Abattement } \\
\text { DCO } \\
(\%)\end{array}$ & $\begin{array}{c}\text { Concentration } \\
\mathrm{N}-\mathrm{NH}_{4} \text { initiale } \\
\left(\mathrm{mg} \cdot \mathrm{L}^{-1}\right)\end{array}$ & $\begin{array}{c}\text { Abattement } \\
\text { N-NH } \\
(\%)\end{array}$ & $\begin{array}{c}\text { Densité de courant } \\
\left(\mathbf{m A} \cdot \mathbf{c m}^{-2}\right) / \\
\text { intensité (A) }\end{array}$ & Références \\
\hline Graphite & Acier & 0,6 & 8 & 240 & $4100-5000$ & 21 & $2100-3000$ & 11 & $75 \mathrm{~mA} \cdot \mathrm{cm}^{-2}$ & CHIANG et al., 1995 \\
\hline $\mathrm{PbO}_{2} / \mathrm{Ti}$ & Acier & 0,6 & 8 & 240 & $4100-5000$ & 27,4 & $2100-3000$ & 33,1 & $75 \mathrm{~mA} \cdot \mathrm{cm}^{-2}$ & CHIANG et al., 1995 \\
\hline $\mathrm{Ru}-\mathrm{Ti}(\mathrm{DSA})^{\mathrm{a}}$ & Acier & 0,6 & 8 & 240 & $4100-5000$ & 29 & $2100-3000$ & 36,1 & $75 \mathrm{~mA} \cdot \mathrm{cm}^{-2}$ & CHIANG et al., 1995 \\
\hline $\mathrm{Sn}-\mathrm{Pb}-\mathrm{Ru}$ & Acier & 0,6 & 8 & 240 & $4100-5000$ & 30,3 & $2100-3000$ & 37,6 & $75 \mathrm{~mA} \cdot \mathrm{cm}^{-2}$ & CHIANG et al., 1995 \\
\hline $\mathrm{BDD}^{\mathrm{b}}$ & Acier inox & - & 8,35 & 480 & - & - & 1934 & 80 & $60 \mathrm{~mA} \cdot \mathrm{cm}^{-2}$ & CABEZA et al., 2007 \\
\hline BDD & Acier inox & - & 8,35 & 360 & - & - & 1934 & $>98$ & $90 \mathrm{~mA} \cdot \mathrm{cm}^{-2}$ & CABEZA et al., 2007 \\
\hline $\mathrm{Al}$ & $\mathrm{Cu}$ & 2 & $8,2-8,5$ & 20 & 1207 & 32,5 & - & - & $45,8 \mathrm{~mA} \cdot \mathrm{cm}^{-2}$ & TSAI et al., 1997 \\
\hline $\mathrm{Fe}$ & $\mathrm{Cu}$ & 2 & $8,2-8,5$ & 20 & 1134 & 36,2 & - & - & $73,7 \mathrm{~mA} \cdot \mathrm{cm}^{-2}$ & TSAI et al., 1997 \\
\hline $\mathrm{Ti} / \mathrm{Pt}$ & Acier inox & 6 & 6,1 & 300 & 60000 & 53 & 1200 & 100 & $33-54 \mathrm{~mA} \cdot \mathrm{cm}^{-2}$ & VLYSSIDES et al., 2003 \\
\hline Graphite & Graphite & 0,6 & 9,1 & 200 & 1414 & 68 & - & - & $79,9 \mathrm{~mA} \cdot \mathrm{cm}^{-2}$ & BASHIR et al., 2009 \\
\hline Acier inox & Acier inox & 2 & 7,5 & 10080 & 4314 & 65 & 2296 & 60,7 & 1,05-0,6 A & RADA et al., 2013 \\
\hline $\mathrm{Ti} / \mathrm{RuO}_{2}-\mathrm{IrO}_{2}$ & Acier inox & 0,2 & 8,85 & 90 & 560 & 66,2 & 520 & 98,4 & $47 \mathrm{~mA} \cdot \mathrm{cm}^{-2}$ & ZHANG et al., 2011 \\
\hline $\mathrm{BDD}$ & BDD & 10 & 5 & 240 & 3385 & 19 & - & - & $6,3 \mathrm{~A}$ & ANGLADA et al., 2011 \\
\hline $\mathrm{Ti} / \mathrm{Pt}$ & Acier inox & 0,2 & 9 & 60 & 2740 & 63,5 & 135 & 80 & $40 \mathrm{~mA} \cdot \mathrm{cm}^{-2}$ & ALOUI et al., 2009 \\
\hline $\mathrm{Ti} / \mathrm{Pt}$ & Acier inox & 0,2 & 9 & 60 & 2740 & 39 & - & - & $15 \mathrm{~mA} \cdot \mathrm{cm}^{-2}$ & ALOUI et al., 2009 \\
\hline
\end{tabular}

${ }^{a}$ DSA : oxydes de titane et de ruthénium recouverts de titane

bBDD : diamant dopé au bore

${ }^{\mathrm{C}} \mathrm{DCO}:$ demande chimique en oxygène

Tableau 4. Résumé des résultats de quelques études sur le traitement de lixiviats de sites d'enfouissement par électro-Fenton.

Table 4. Summary of results of some studies on landfill leachate treatment by electro-Fenton.

\begin{tabular}{|c|c|c|c|c|c|c|c|c|c|c|c|}
\hline Anode & Cathode & Type de lixiviat & $\begin{array}{l}\text { Volume de } \\
\text { travail } \\
\text { (L) }\end{array}$ & $\begin{array}{l}\mathrm{pH} \mathrm{de} \\
\text { travail }\end{array}$ & $\begin{array}{c}\text { Durée de } \\
\text { traitement } \\
(\text { min) }\end{array}$ & $\begin{array}{c}\text { Concentration } \\
\text { DCO initiale } \\
\left(\mathbf{m g} \cdot \mathrm{L}^{-1}\right)\end{array}$ & $\begin{array}{c}\text { Abattement } \\
\text { DCO } \\
(\%)\end{array}$ & $\begin{array}{c}\text { Concentration } \\
\mathrm{H}_{2} \mathrm{O}_{2} \\
\left(\mathbf{m o l} \cdot \mathrm{L}^{-1}\right)\end{array}$ & $\begin{array}{c}\text { Ratio } \\
\text { molaire } \\
\mathrm{H}_{2} \mathrm{O}_{2} / \mathrm{Fe}^{2+} \\
\end{array}$ & $\begin{array}{c}\text { Densité de courant } \\
\left(\mathbf{m A} \cdot \mathbf{c m}^{-2}\right) / \\
\text { intensité }(\mathbf{A})\end{array}$ & Références \\
\hline $\mathrm{Pt}$ & Feutre de carbone & Lixiviat brut & 0,4 & 3 & 480 & 10200 & 78 & - & - & $7,6 \mathrm{~mA} \cdot \mathrm{cm}^{-2}$ & TRABELSI et al., 2012 \\
\hline $\mathrm{Ti}$ & $\begin{array}{c}\mathrm{Ti} / \mathrm{RuO}_{2}-\mathrm{IrO}_{2} \\
-\mathrm{SnO}_{2}-\mathrm{TiO}_{2}\end{array}$ & Lixiviat brut & 0,8 & 3 & 40 & 2720 & 62 & 0,17 & 6 & $1 \mathrm{~A}$ & ZHANG et al., 2012a \\
\hline $\begin{array}{l}\mathrm{Ti} / \mathrm{RuO}_{2} \\
-\mathrm{IrO}_{2}\end{array}$ & $\mathrm{Ti} / \mathrm{RuO}_{2}-\mathrm{IrO}_{2}$ & Lixiviat brut & 0,2 & 3 & 75 & 5000 & 87,2 & 0,34 & 12 & $42 \mathrm{~mA} \cdot \mathrm{cm}^{-2}$ & ZHANG et al., 2006 \\
\hline $\mathrm{Al}$ & $\mathrm{Al}$ & Lixiviat brut & 0,5 & 3 & 43 & $2820-3150$ & 94 & - & 1 & $49 \mathrm{~mA} \cdot \mathrm{cm}^{-2}$ & MOHAJERI et al., 2010 \\
\hline $\mathrm{Fe}$ & $\mathrm{Fe}$ & Lixiviat coagulé & 1 & 4 & 30 & 950 & 68,3 & 0,022 & - & $113,6 \mathrm{~mA} \cdot \mathrm{cm}^{-2}$ & LIN et CHANG, 2000 \\
\hline $\mathrm{Fe}$ & $\mathrm{Fe}$ & Lixiviat brut & 0,5 & 3 & 20 & 2350 & 72 & 0,058 & - & $3 \mathrm{~A}$ & ATMACA, 2009 \\
\hline $\mathrm{Fe}$ & $\mathrm{Fe}$ & Lixiviat brut & 0,9 & 3,4 & 60 & 11000 & 74,21 & 0,147 & - & $20 \mathrm{~mA} \cdot \mathrm{cm}^{-2}$ & ORKUN et KULEYIN, 2012 \\
\hline $\begin{array}{l}\mathrm{Ti} / \mathrm{RuO}_{2} \\
-\mathrm{IrO}_{2}\end{array}$ & Acier inox & Lixiviat brut & 0,8 & 3 & 140 & $3424-3680$ & $\approx 82$ & 0,34 & 12 & $19,2 \mathrm{~mA} \cdot \mathrm{cm}^{-2}$ & ZHANG et al., 2012b \\
\hline $\mathrm{Fe}$ & $\mathrm{Fe}$ & Lixiviat brut & 1 & 3 & 20 & 2350 & 70 & 0,058 & - & $2 \mathrm{~A}$ & ALTIN, 2008 \\
\hline
\end{tabular}


l'augmentation du $\mathrm{pH}$ sont : l'autodécomposition du peroxyde d'hydrogène en $\mathrm{H}_{2} \mathrm{O}$ et $\mathrm{O}_{2}$ (DENG et ENGLEHARDT, 2006), le piégeage des radicaux hydroxyles par les ions carbonates et bicarbonates $\left(\mathrm{CO}_{3}^{2-}\right.$ et $\mathrm{HCO}_{3}^{-}$) (DENG et ENGLEHARDT, 2006) et la diminution du potentiel d'oxydation du radical hydroxyle (KIM et VOGELPOHL, 1998). Dans les mêmes conditions opératoires, ZHANG et al. (2012a) ont observé des taux d'abattement de la DCO de $17 \%$ et $58 \%$ avec des $\mathrm{pH}$ respectifs 8 et 3 . Cependant, en travaillant avec un $\mathrm{pH} 5$, le taux d'abattement est de $55 \%$, mais la vitesse de réaction est moins élevée par rapport à l'essai réalisé à $\mathrm{pH} 3$. Cela montre l'influence du $\mathrm{pH}$ sur le traitement d'EF. Selon ATMACA (2009) une élimination de $64 \%$ de la DCO a été obtenue à $\mathrm{pH} 3$ tandis que l'abattement était aux alentours de $10 \%$ à pH 2 (limite de l'étendue optimale).

D’une façon générale, l'augmentation du courant électrique à un effet positif sur l'efficacité du procédé EF. Elle permet de former plus de peroxyde d'hydrogène à partir de la réduction de l' $\mathrm{O}_{2}$ dissous. Les vitesses de génération du fer(II) à l'anode sacrificielle et la régénération cathodique du fer(II) sont proportionnelles à l'augmentation du courant (NIDHEESH et GANDHIMATHI, 2012; ATMACA, 2009). Néanmoins, une forte valeur de courant peut ne pas avoir un effet significatif sur les performances du procédé (ATMACA, 2009; ZHANG et al., 2006). ATMACA (2009) a observé une limite d'abattement de la DCO pour une valeur d'intensité de courant supérieure à $2 \mathrm{~A}$ avec une anode en fer. En effet, au-delà de cette intensité, il peut se former des réactions parasites telles que l'oxydation du peroxyde d'hydrogène en oxygène et la réduction des $\mathrm{H}^{+}$ en $\mathrm{H}_{2}$ ayant comme conséquence la réduction du potentiel de formation des radicaux hydroxyles et la diminution de l'acidité du milieu. Une valeur optimale d'intensité de courant doit être précieusement déterminée afin d'avoir un maximum d'abattement tout en minimisant la consommation électrique et la production de résidus métalliques après la phase de neutralisation. Les densités de courant optimales obtenues par ATMACA (2009) et ZHANG et al. (2006) lors du traitement des lixiviats par EF sont respectivement 100 et $42 \mathrm{~mA} \cdot \mathrm{cm}^{-2}$.

La quantité de réactifs et la proportion molaire entre le peroxyde d'hydrogène et les ions ferreux ont une influence majeure sur l'efficacité de la réaction de Fenton. L'excès ou la carence de l'un des deux réactifs peut avoir un impact négatif sur le traitement (DENG et ENGLEHARDT, 2006). Des concentrations faibles en peroxyde et en fer(II) limitent la production des radicaux hydroxyles responsables de la dépollution. En revanche, les fortes concentrations en ions ferreux favorisent une surproduction de boues. L'excès de peroxyde d'hydrogène entrâne la flottation des boues via les bulles d'oxygène issues de la décomposition du peroxyde (DENG et ENGLEHARDT, 2006). La quantité de réactif utilisée est directement liée à la charge de DCO de l'effluent à traiter (DENG et ENGLEHARDT, 2006). En fixant un ratio molaire $\mathrm{H}_{2} \mathrm{O}_{2} / \mathrm{Fe}(\mathrm{II})$ de 12 , ZHANG et al. (2006) ont observé une augmentation linéaire de l'élimination de la DCO avec des ajouts croissants de peroxyde. Cependant, le taux d'abattement de la DCO stagne au-dessus d'une concentration de $0,34 \mathrm{~mol} \cdot \mathrm{L}^{-1}$ de peroxyde. La même expérience a mis en évidence l'amélioration de la qualité de traitement avec l'augmentation de la quantité de fer(II) jusqu'à une valeur limite. Ces résultats prouvent l'existence d'un ratio $\mathrm{H}_{2} \mathrm{O}_{2}$ / $\mathrm{Fe}(\mathrm{II})$ optimal pour le traitement d'électro-Fenton. Une grande divergence existe sur la valeur du ratio. La quantité de réactifs utilisée par charge de DCO éliminée varie selon les caractéristiques des lixiviats et les méthodes de détermination (DENG et ENGLEHARDT, 2006). Le ratio $\mathrm{H}_{2} \mathrm{O}_{2} / \mathrm{Fe}(\mathrm{II})$ optimal défini par ZHANG et al. (2012a) est de 1 tandis que ZHANG et al. (2006) et MOHAJERI et al. (2010) ont travaillé avec des ratios respectifs de 12 et 1 .

La distance interélectrodes a été étudiée par ZHANG et al. (2012a) lors du traitement d'un lixiviat mature. Ils ont observé une distance interélectrodes optimale entre 1 et $2,5 \mathrm{~cm}$. Dans le cas de certaines configurations, les ions ferreux sont régénérés par réduction cathodique des ions ferriques, ainsi une distance interélectrodes très faible favoriserait l'oxydation anodique des ions ferreux (ZHANG et al., 2006). ATMACA (2009) a conclu que la distance interélectrodes n'a pas d'influence significative sur l'efficacité du traitement, cependant; une grande distance entre les électrodes peut entraîner une consommation énergétique supplémentaire.

Il est à noter que le procédé d'EF n'est pas efficace pour le traitement de l'azote ammoniacal. Les radicaux hydroxyles n'oxydent pas efficacement l'ammonium. Les faibles pourcentages d'abattement de l'azote ammoniacal obtenu à des $\mathrm{pH}$ basiques sont attribués aux phénomènes de coagulation et de stripage (ATMACA, 2009).

\section{PROCÉDÉD’ÉLECTROCOAGULATION}

Le procédé d'électrocoagulation (EC) est issu du procédé de coagulation chimique qui utilise des réactifs chimiques pour la coagulation des colloïdes présents dans les eaux. Pendant l'EC, les agents coagulants $\left(\mathrm{Fe}^{2+}\right.$ et $\left.\mathrm{Al}^{3+}\right)$ sont générés in situ de façon électrochimique en appliquant un courant électrique sur une anode sacrificielle en fer ou en aluminium (équations 16 et 17).

$$
\begin{aligned}
& \mathrm{Fe} \rightarrow \mathrm{Fe}^{2+}+2 \mathrm{e}^{-} \\
& \mathrm{Al} \rightarrow \mathrm{Al}^{3+}+3 \mathrm{e}^{-}
\end{aligned}
$$


Les ions produits réagissent avec les ions hydroxydes $\left(\mathrm{OH}^{-}\right)$ présents dans la solution pour former des hydroxydes de fer ou d'aluminium (équations 18 et 19) (DROGUI et al., 2007).

$$
\begin{aligned}
& \mathrm{Fe}^{2+}+\mathrm{OH}^{-} \rightarrow \mathrm{Fe}(\mathrm{OH})_{2} \\
& \mathrm{Al}^{3+}+\mathrm{OH}^{-} \rightarrow \mathrm{Al}(\mathrm{OH})_{3}
\end{aligned}
$$

À la cathode, il y a formation du gaz $\mathrm{H}_{2}$ qui entraîne la flottation des résidus métalliques (Équation 20) (CHEN, 2004).

$$
2 \mathrm{H}_{2} \mathrm{O}+2 \mathrm{e}^{-} \rightarrow \mathrm{H}_{2}+2 \mathrm{OH}^{-}
$$

Le mécanisme d'EC dépend fortement de la nature chimique du milieu et plus particulièrement de sa conductivité (MOLLAH et al., 2001). Les hydroxydes métalliques formés lors des réactions, exprimées par les équations 18 et 19, s'impliquent dans des réactions de condensation ou de polymérisation. Ce qui conduit à la formation d'un précipité vert pour les hydroxydes de fer et d'une substance gélatineuse blanche pour les hydroxydes d'aluminium (DROGUI et al., 2007). Les réactions de polymérisation peuvent être décrites par les équations 21 et 22 . Les complexes polymériques formés sont capables d'éliminer les polluants dissous et insolubles par des phénomènes d'adsorption, de complexation ou de précipitation (DROGUI et al., 2007).

$$
\begin{aligned}
& 2 \mathrm{Al}(\mathrm{OH})_{3} \rightarrow(\mathrm{OH})_{2} \mathrm{Al}-\mathrm{O}-\mathrm{Al}(\mathrm{OH})_{2}+\mathrm{H}_{2} \mathrm{O} \\
& 2 \mathrm{Fe}(\mathrm{OH})_{2} \rightarrow(\mathrm{OH}) \mathrm{Fe}-\mathrm{O}-\mathrm{Fe}(\mathrm{OH})+\mathrm{H}_{2} \mathrm{O}
\end{aligned}
$$

Les ions de fer et d'aluminium peuvent générer également différentes espèces coagulantes de charge positive qui provoquent la déstabilisation et l'agglomération des particules et colloïdes chargés négativement. Par exemple, l'hydrolyse des ions $\mathrm{Al}^{3+}$ génère, sur une large gamme de valeurs de $\mathrm{pH}$, des espèces comme $\mathrm{Al}\left(\mathrm{H}_{2} \mathrm{O}\right)_{6}{ }_{6}^{3+}, \mathrm{Al}\left(\mathrm{H}_{2} \mathrm{O}\right)_{5}{ }^{2+}$ et $\mathrm{Al}\left(\mathrm{H}_{2} \mathrm{O}\right)_{4}{ }_{4}^{2+}$. L'hydrolyse de ces dernières conduit à la formation de différentes espèces monomériques ou polymériques telles que $\mathrm{Al}(\mathrm{OH})^{2+}$, $\mathrm{Al}(\mathrm{OH})_{2}{ }^{+}, \mathrm{Al}_{2}(\mathrm{OH})_{2}{ }_{2+}^{4+}, \mathrm{Al}(\mathrm{OH})_{4}, \mathrm{Al}_{6}(\mathrm{OH})_{15}{ }^{3+}, \mathrm{Al}_{7}(\mathrm{OH})_{17}{ }^{4+}$, etc. (MOLLAH et al., 2004). Un phénomène similaire est observé avec l'hydrolyse des ions ferriques. Selon le $\mathrm{pH}$ du milieu, il est possible de former l'espèce monomérique $\mathrm{Fe}(\mathrm{OH})_{3}$ et des espèces polymériques complexes comme $\mathrm{Fe}\left(\mathrm{H}_{2} \mathrm{O}\right)_{6}{ }^{3+}$, $\mathrm{Fe}\left(\mathrm{H}_{2} \mathrm{O}\right)_{5}(\mathrm{OH})^{2+}, \quad \mathrm{Fe}\left(\mathrm{H}_{2} \mathrm{O}\right)_{4}(\mathrm{OH})_{2}^{+}, \quad \mathrm{Fe}_{2}\left(\mathrm{H}_{2} \mathrm{O}\right)_{8}(\mathrm{OH})_{2}^{4+}$ et $\mathrm{Fe}_{2}\left(\mathrm{H}_{2} \mathrm{O}\right)_{6}(\mathrm{OH})_{4}^{4+}$ (MOLLAH et al., 2004).

La combinaison de différents phénomènes de dépollution (électro-oxydation, complexation, précipitation, flottation, coagulation et adsorption) rend ce procédé très efficace pour le traitement des polluants organiques et inorganiques (DROGUI et al., 2007). L'EC est également efficace pour l'élimination des grosses molécules (TSAI et al., 1997). Ces molécules sont largement présentes dans les lixiviats " matures " et sont difficilement biodégradables par une filière biologique. Des études sur le traitement des lixiviats par EC ont été réalisées au cours de ces dernières années et ont des résultats intéressants en termes d'élimination de la DCO et de la couleur (BOUHEZILA et al., 2011; CONTRERAS et al., 2009; ILHAN et al., 2008; LI et al., 2011; MARIAM et NGHIEM, 2010; TSAI et al., 1997). En revanche, l'efficacité de l'EC sur l'élimination de la pollution azotée est relativement faible. Le tableau 5 résume les résultats de quelques études expérimentales portant sur l'EC des lixiviats.

Dans les essais de laboratoire, l'EC est souvent réalisée dans une cellule électrolytique dans laquelle sont plongées les électrodes plates soumises à une différence de potentiel. Le fer, l'aluminium et leurs dérivés sont largement utilisés comme anode soluble pour former les coagulants. Ces deux éléments peuvent également faire office de cathode ou d'autres éléments métalliques comme le cuivre (TSAI et al., 1997). La disposition des électrodes peut se faire selon deux configurations : monopolaire ou bipolaire (DROGUI et al., 2007) (Figure 2). Dans la configuration monopolaire, on a une succession d'anodes et de cathodes reliées respectivement aux bornes positive et négative du générateur. De ce fait, chaque électrode reçoit la même valeur de la tension et une fraction de l'intensité totale. Dans la configuration bipolaire, seules les deux électrodes externes sont reliées aux bornes du générateur. Pour les autres électrodes intermédiaires, il se forme une anode dans une face et une cathode dans l'autre. Les deux configurations sont utilisées en traitement des eaux, cependant la configuration bipolaire est souvent préférée à cause de la simplicité des connexions et des faibles dissipations énergétiques dans le circuit externe (DROGUI et al., 2007).

Parmi les paramètres qui influencent le traitement d'EC, on peut citer l'intensité appliquée, le type d'électrode, le $\mathrm{pH}$, la conductivité, la distance interélectrodes et le temps de traitement (KHANDEGAR et SAROHA, 2013).

Selon la loi de Faraday, l'intensité appliquée est directement proportionnelle à la quantité de coagulants produits à l'anode. Une augmentation du courant électrique favorise les réactions décrites par les équations 16 et 17 , induisant une meilleure élimination des polluants. Une augmentation de l'intensité est également favorable à la formation du dihydrogène à la cathode (Réaction 20) permettant ainsi la flottation des hydroxydes métalliques vers la surface du réacteur. L'effet de la densité de courant sur l'EC des lixiviats a été étudié par quelques auteurs. TSAI et al. (1997) ont observé une augmentation de la vitesse et du taux d'élimination de la DCO en faisant varier la tension entre 2 et $15 \mathrm{~V}$. Ils ont également observé que l'utilisation d'une anode en Fe donne de meilleurs résultats par rapport 
Tableau 5. Résumé des résultats de quelques études faites sur l'électrocoagulation des lixiviats de sites d'enfouissement.

Table 5. Summary of results of some studies on electrocoagulation of landfill leachates.

\begin{tabular}{|c|c|c|c|c|c|c|c|c|c|c|c|}
\hline Anode & Cathode & $\begin{array}{l}\text { Volume } \\
\text { de travail } \\
(\mathrm{L})\end{array}$ & pH & $\begin{array}{c}\text { Durée de } \\
\text { traitement } \\
(\mathrm{min})\end{array}$ & $\begin{array}{c}\text { Tension }(\mathrm{V}) / \\
\text { densité de courant } \\
\left(\mathrm{mA} \cdot \mathrm{cm}^{-2}\right)\end{array}$ & $\begin{array}{c}\text { Concentration } \\
\text { DCO }^{\mathrm{a}} \text { initiale } \\
\left(\mathrm{mg} \cdot \mathrm{L}^{-1}\right)\end{array}$ & $\begin{array}{l}\text { Abattement } \\
\text { DCO } \\
(\%)\end{array}$ & $\begin{array}{c}\text { Concentration } \\
\mathrm{NH}_{4} \text { initiale } \\
\left(\mathbf{m g} \cdot \mathbf{L}^{-1}\right)\end{array}$ & $\begin{array}{c}\text { Abattement } \\
\mathrm{NH}_{4} \\
(\%)\end{array}$ & $\begin{array}{c}\text { Distance } \\
\text { interélectrodes } \\
(\mathbf{c m})\end{array}$ & Références \\
\hline $\mathrm{Al}$ & $\mathrm{Al}$ & 0,5 & 8 & 100 & $12 \mathrm{~V}$ & 16464 & 62,7 & - & - & 6,5 & JOTIN et al., 2012 \\
\hline $\mathrm{Fe}$ & $\mathrm{Fe}$ & 1 & $6,4-7,3$ & 90 & $4,96 \mathrm{~mA} \cdot \mathrm{cm}^{-2}$ & 2566 & 49,8 & 386 & 38,6 & 1 & LI et al., 2011 \\
\hline $\mathrm{Fe}$ & $\mathrm{Fe}$ & 1 & 6,5 & 30 & $2,98 \mathrm{~mA} \cdot \mathrm{cm}^{-2}$ & 2566 & 32,7 & 386 & 24,8 & 1 & LI et al., 2011 \\
\hline $\mathrm{Al}$ & $\mathrm{Al}$ & 1 & 6,5 & 30 & $2,98 \mathrm{~mA} \cdot \mathrm{cm}^{-2}$ & 2566 & 21 & 386 & 20,8 & 1 & LI et al., 2011 \\
\hline $\mathrm{Al}$ & $\mathrm{Al}$ & 0,5 & 8,2 & 30 & $63,1 \mathrm{~mA} \cdot \mathrm{cm}^{-2}$ & 12860 & 56 & 2240 & 14 & 6,5 & ILHAN et al., 2008 \\
\hline $\mathrm{Fe}$ & $\mathrm{Fe}$ & 0,5 & 8,2 & 30 & $63,1 \mathrm{~mA} \cdot \mathrm{cm}^{-2}$ & 12860 & $\approx 52$ & 2240 & 11 & 6,5 & ILHAN et al., 2008 \\
\hline $\mathrm{Al}$ & $\mathrm{Al}$ & 0,5 & $7,6-8,9$ & 30 & $50 \mathrm{~mA} \cdot \mathrm{cm}^{-2}$ & $28200-34200$ & 70 & - & - & 2,8 & BOUHEZILA et al., 2011 \\
\hline $\mathrm{Fe}$ & $\mathrm{Fe}$ & 0,5 & $7,6-8,9$ & 30 & $50 \mathrm{~mA} \cdot \mathrm{cm}^{-2}$ & $28200-34200$ & $\approx 68$ & - & - & 2,8 & BOUHEZILA et al., 2011 \\
\hline $\mathrm{Al}$ & $\mathrm{Cu}$ & 2 & $8,2-8,5$ & 20 & $25 \mathrm{~mA} \cdot \mathrm{cm}^{-2}$ & 2247 & 39 & - & - & 2 & TSAI et al., 1997 \\
\hline $\mathrm{Fe}$ & $\mathrm{Cu}$ & 2 & $8,2-8,5$ & 20 & $26,1 \mathrm{~mA} \cdot \mathrm{cm}^{-2}$ & 2107 & 44,5 & - & - & 2 & TSAI et al., 1997 \\
\hline $\mathrm{Al}$ & $\mathrm{Al}$ & - & 8 & 60 & $20 \mathrm{~mA} \cdot \mathrm{cm}^{-2}$ & 9800 & 60,5 & 3710 & 24,3 & - & KABUK et al., 2014 \\
\hline $\mathrm{Fe}$ & $\mathrm{Fe}$ & 0,15 & 8,6 & 30 & $5 \mathrm{~V}$ & 12650 & 33 & - & - & 2,3 & NORMA et al., 2012b \\
\hline $\mathrm{Al}$ & $\mathrm{Al}$ & 0,8 & $6,61-7$ & 30 & $15,9 \mathrm{~mA} \cdot \mathrm{cm}^{-2}$ & 6200 & 45 & - & - & 1,6 & TOP et al., 2011 \\
\hline $\mathrm{Fe}$ & $\mathrm{Fe}$ & 0,9 & 6,54 & 180 & $30 \mathrm{~mA} \cdot \mathrm{cm}^{-2}$ & 11000 & 65,8 & - & - & 0,9 & ORKUN et KULEYIN, 2012 \\
\hline
\end{tabular}

${ }^{\mathrm{a}} \mathrm{DCO}$ : demande chimique en oxygène 

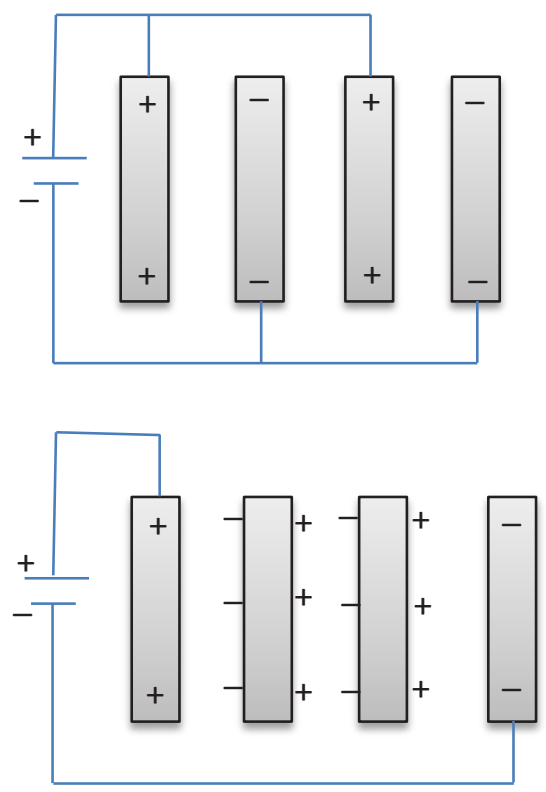

Figure 2. Configurations monopolaire (a) et bipolaire des électrodes (b). Monopolar (a) and bipolar (b) electrode configurations.

à une anode en $\mathrm{Al}$ à des faibles valeurs de tension. Selon LI et al. (2011), avec l'augmentation du courant, les bulles de dihydrogène $\mathrm{H}_{2}$ produites à la cathode deviennent plus denses et leur taille diminue. Ce phénomène rend l'électro-flottation plus efficace. D'autres expériences réalisées par ILHAN et al. (2008) et BOUHEZILA et al. (2011) sur des lixiviats ont mis en évidence l'augmentation des performances épuratoires avec l'augmentation de la densité de courant. Cependant, une forte valeur de la tension électrique peut réduire les taux d'abattement de la DCO (JOTIN et al., 2012). Ce phénomène peut être dû à la réaction d'hydrolyse de l'eau en $\mathrm{O}_{2}$ qui se produit à des valeurs élevées d'intensités de courant et qui rentre en compétition avec la réaction de dissolution anodique (CANIZARES et al., 2005a).

Étant donné que les réactions d'hydrolyse des ions fer et aluminium dépendent $\mathrm{du} \mathrm{pH}$, ce dernier a un rôle important dans un procédé d'EC. Selon une étude de JOTIN et al. (2012), l'élimination de la DCO varie de 59 à $73 \%$ pour une étendue de $\mathrm{pH}$ initial du lixiviat allant de 4 à 8. L'étude a montré qu'une correction de $\mathrm{pH}$ du lixiviat n'est pas nécessaire, car le $\mathrm{pH}$ initial est situé dans cette plage. FERNANDES et al. (2014) ont également conclu que le $\mathrm{pH}$ initial du lixiviat étudié convient comme $\mathrm{pH}$ optimal avec l'utilisation d'une électrode en fer. Cependant, les valeurs de $\mathrm{pH}$ très acides $(<3,9)$ ou très basiques $(>10)$ diminuent les performances du procédé d'EC avec une chute brutale du taux d'élimination de la DCO (LI et al., 2011). En fait, à des valeurs de $\mathrm{pH}$ très basiques $(\mathrm{pH}>$ 10), la charge électrostatique de la surface des colloïdes est modifiée, ce qui empêche leur déstabilisation (DROGUI et al.,
2008). De plus, certains hydroxydes de fer ou d'aluminium formés à des $\mathrm{pH}$ basiques portent des charges négatives inefficaces pour l'EC (DROGUI et al., 2008). Les $\mathrm{pH}$ limites de solubilité des hydroxydes de fer $\left[\mathrm{Fe}(\mathrm{OH})_{2}\right.$ et $\left.\mathrm{Fe}(\mathrm{OH})_{3}\right]$ et des hydroxydes d'aluminium $\left[\mathrm{Al}(\mathrm{OH})_{3}\right]$ sont respectivement de 5,5 et 4 . Ce qui explique les meilleures performances de l'Al à des $\mathrm{pH}$ légèrement acides par rapport au fer (ASSELIN, 2007; MORENO-CASILLAS et al., 2007). La valeur du pH initial augmente après traitement par EC (LI et al., 2011). Cette hausse est principalement attribuée à la production d'ions hydroxydes lors de la réduction cathodique de l'eau (Équation 5) (DROGUI et al., 2008).

Le type d'électrode ( $\mathrm{Fe}$ ou $\mathrm{Al}$ ) utilisé peut avoir un impact sur la qualité du lixiviat traité. Selon BOUHEZILA et al. (2011), l'anode en $\mathrm{Al}$ est plus efficace que celle en fer pour le traitement de la couleur, de la turbidité et du $\mathrm{NH}_{4}$ présents dans les lixiviats. En revanche, le taux d'abattement de la DCO n'est pas significativement influencé par le type d'électrode (BOUHEZILA et al., 2011). Les mêmes résultats en termes de DCO et de $\mathrm{NH}_{4}$ ont été obtenus par ILHAN et al. (2008) en présence d'électrodes en fer et en aluminium dans les mêmes conditions opératoires. Les deux études précitées ont été réalisées à des densités de courant de 50 et $63 \mathrm{~mA} \cdot \mathrm{cm}^{-2}$ respectivement. En utilisant une densité de courant plus faible $\left(2,98 \mathrm{~mA} \cdot \mathrm{cm}^{-2}\right)$, LI et al. (2011) ont trouvé que l'anode en Fe permet d'éliminer plus de DCO que celle en Al. TSAI et al. (1997) ont également montré que l'utilisation d'une anode en Fe donne de meilleurs résultats par rapport à une anode en $\mathrm{Al}$ à des faibles valeurs de tension. D'après LI et al. (2011), les hydroxydes de fer décantent mieux que les hydroxydes d'aluminium. Ceci peut être expliqué du fait que le fer présente une masse molaire plus élevée que l'Al. Le volume des résidus métalliques générés est deux fois plus élevé en changeant l'électrode de Fe par une électrode d'Al selon une étude de BOUHEZILA et al. (2011). Selon nos connaissances actuelles, l'effet des configurations monopolaire et bipolaire sur l'EC des lixiviats n'a pas encore été étudié.

L'augmentation de la conductivité permet d'optimiser la consommation énergétique et de réduire le temps de traitement (ILHAN et al., 2008; KHANDEGAR et SAROHA, 2013). Vu la valeur élevée de la conductivité des lixiviats, l'ajout d'électrolyte, dans le but d'augmenter la conductivité, n'est pas nécessaire dans la plupart des cas (JOTIN et al., 2012). Le $\mathrm{NaCl}$ est souvent utilisé comme électrolyte, mais un fort dosage peut entraîner la réaction entre les ions chlorures et les hydroxydes métalliques qui forment des composés chlorés tels que $\mathrm{Al}(\mathrm{OH})_{2} \mathrm{Cl}$ et $\mathrm{Al}(\mathrm{OH}) \mathrm{Cl}_{2}$ non favorables à la coagulation (WANG et al., 2009a). Dans les études effectuées au laboratoire, le taux de dépollution augmente avec le temps d'hydrolyse avant de plafonner à des valeurs allant de 30 à $100 \mathrm{~min}$ de temps de traitement selon l'intensité de courant appliquée. Un temps de traitement long conduit à une consommation 
d'énergie élevée (LI et al., 2011). Étant donné que la chute ohmique est proportionnelle à la distance interélectrodes, l'augmentation ou la réduction de cette distance peut influencer les performances du traitement. BOUHEZILA et al. (2011) ont observé une variation de $10 \%$ du taux d'abattement de la DCO en faisant variant la distance interélectrodes de 0,5 à $2,8 \mathrm{~cm}$. Une distance interélectrodes très large peut aboutir à une consommation supplémentaire en énergie. La distance interélectrodes varie entre 1 et $6,5 \mathrm{~cm}$ dans les études consultées. Le procédé d'EC présente un net avantage pour la dépollution des métaux. Une étude réalisée par DERMENTZIS et al. (2011) sur l'électrocoagulation d'un effluent industriel chargé en $\mathrm{Ni}, \mathrm{Cu}, \mathrm{Zn}$ et $\mathrm{Cr}$ à des concentrations de $50 \mathrm{mg} \cdot \mathrm{L}^{-1}$ a révélé des taux d'abattements supérieurs à $97 \%$ pour le $\mathrm{Ni}$, le $\mathrm{Cu}$ et le $\mathrm{Zn}$ et supérieurs à $80 \%$ pour le Cr. Une autre étude menée par MEUNIER et al. (2006) a mis en évidence l'efficacité du procédé d'EC pour l'élimination du $\mathrm{Pb}$ contenu dans des lixiviats de sols contaminés. Des taux d'élimination supérieurs à $94 \%$ ont été enregistrés pour des concentrations initiales en $\mathrm{Pb}$ variant de 250 à $2000 \mathrm{mg} \cdot \mathrm{L}^{-1}$.

\section{AVANTAGES ET INCONVÉNIENTS DES PROCÉDÉS ÉLECTROCHIMIQUES}

Les procédés électrochimiques (EO, EC et $\mathrm{EF}$ ) ont l'avantage d'éliminer à la fois la pollution organique et la couleur des lixiviats. Le tableau 6 récapitule les principaux avantages et inconvénients des trois procédés électrochimiques. Grâce à la combinaison des réactions directes et indirectes, l'électrooxydation est en mesure de dégrader à la fois les composés organiques et inorganiques comme le $\mathrm{NH}_{4}$. Contrairement à l'EF, l'EO permet d'opérer dans une étendue de $\mathrm{pH}$ initial des lixiviats allant de 6 à 8 . Toutefois, l'oxydation indirecte requiert des concentrations élevées en chlorures pour favoriser la formation des intermédiaires réactifs (CHEN, 2004). Il existe également un risque de formation de composés organochlorés toxiques liés aux fortes concentrations en ions chlorures (CHIANG et al., 1995). L'EF est particulièrement reconnue pour ses bonnes performances en termes d'élimination de la DCO et d'augmentation de la biodégradabilité de l'effluent. En revanche, il nécessite un $\mathrm{pH}$ de traitement acide et un ajout de peroxyde d'hydrogène ou d'ions ferriques dans certaines dispositions (QIANG et al., 2003). Son inefficacité à éliminer l'azote ammoniacal fait que cette technologie est toujours couplée à un autre procédé capable de traiter le $\mathrm{NH}_{4}$. À propos de l'EC, la combinaison des processus électrochimiques et physico-chimiques entraîne une élimination efficace de la turbidité et des composés organiques ayant une masse moléculaire élevée. L'EC produit également moins de résidus métalliques comparativement aux procédés de coagulation chimique (ILHAN et al., 2008). Cependant, tout comme
l'EF, la gestion des boues chargées en résidus métalliques est une problématique qui doit être prise en compte. Lazote ammoniacal est faiblement éliminé pendant l'EC, certains auteurs avancent que l'élimination du $\mathrm{NH}_{4}$ observée lors de l'EC est liée au phénomène de stripping qui transforme le $\mathrm{NH}_{4}$ en $\mathrm{NH}_{3}$ gazeux (ATMACA, 2009; ILHAN et al., 2008).

\section{COMBINAISON DES PROCÉDÉS ÉLECTROCHIMIQUESETBIOLOGIQUES POUR LE TRAITEMENT DES LIXIVIATS DE SITES D'ENFOUISSEMENT}

Les procédés biologiques sont largement utilisés pour le traitement des lixiviats. On rencontre dans la littérature, différentes technologies biologiques appliquées au traitement des lixiviats : lagunage (ROBINSON et GRANTHAM, 1988), boue activée (HOSOMI et al., 1989), lit bactérien (BOUCHER et al., 2010; MARTIENSSEN et al., 1995), biodisque (HENDERSON et ATWATER, 1995), bioréacteur à membrane (MBR) (BODZEK et al., 2006), réacteur biologique discontinu (SBR) (YING et al., 1986), etc. Les procédés biologiques présentent comme principaux atouts l'efficacité de traiter les polluants organiques biodégradables de façon rentable. Ils sont aussi efficaces pour le traitement des lixiviats "jeunes " qui sont caractérisés par un rapport de biodégradabilité (DBO/DCO) élevé. Cependant, leur utilisation est limitée pour l'épuration des lixiviats " matures " qui présentent un grand pourcentage de composés bioréfractaires (XIAO et al., 2013). Concernant les procédés électrochimiques, leur efficacité vis-à-vis de la pollution bioréfractaire est bien connue. En revanche, les coûts énergétiques que requièrent ces procédés peuvent être un frein pour des applications à l'échelle industrielle. Face à ce problème, le couplage des procédés biologiques et électrochimiques a été étudié depuis quelques années. Cette combinaison est techniquement et économiquement intéressante dans la mesure où elle permet de réduire les coûts d'opération par l'intégration de la filière biologique (WANG et al., 2009b; DROGUI et al., 2007) et d'améliorer les taux d'abattement des composés réfractaires via les procédés électrochimiques (QUAN et al., 2013; FEKI et al., 2009). La disposition des deux procédés dans la filière de traitement varie selon les cas. Le procédé électrochimique peut être placé en amont du procédé biologique entraînant une dégradation des composés récalcitrants en des composés plus biodégradables (DROGUI et al., 2007; LI et al., 2007). Dans d'autres cas, le procédé électrochimique est placé en aval du procédé biologique dans le but d'oxyder de façon complète les matières organiques résiduelles non biodégradables (DROGUI et al., 2007; QUAN et al., 2013; XIAO et al., 2013). 
Tableau 6. Avantage et inconvénients des différents procédés électrochimiques.

Table 6. Advantages and drawbacks of the different electrochemical processes.

\begin{tabular}{|c|c|}
\hline Avantages & Inconvénients \\
\hline \multicolumn{2}{|c|}{ Électro-oxydation } \\
\hline $\begin{array}{l}\text { - Élimination de la } \mathrm{DCO}^{a} \text { et du } \mathrm{NH}_{4} \\
\text { - } \text { Oxydation directe et indirecte } \\
\text { - } \text { Traitement avec } \mathrm{pH} \text { initial du lixiviat } \\
\text { - Amélioration de la biodégradabilité } \\
\text { - Absence de production de boues }\end{array}$ & $\begin{array}{l}\text { - Formation potentielle de composés organochlorés } \\
\text { - Ajout d'ions chlorures pour augmenter l'oxydation indirecte }\end{array}$ \\
\hline \multicolumn{2}{|c|}{ Électro-Fenton } \\
\hline $\begin{array}{l}\text { - Très bonne efficacité d'élimination des composés organiques } \\
\text { - Bonne amélioration de la biodégradabilité de l'effluent }\end{array}$ & $\begin{array}{l}\text { - Traitement en milieu acide } \\
\text { - Inefficace sur l'élimination du } \mathrm{NH}_{4} \\
\text { - Gestion des résidus métalliques formés } \\
\text { - Ajout de } \mathrm{H}_{2} \mathrm{O}_{2} \text { et } \mathrm{Fe}^{2+} \text { dans certains cas }\end{array}$ \\
\hline \multicolumn{2}{|c|}{ Électrocoagulation } \\
\hline $\begin{array}{l}\text { - Élimination de la DCO et de la couleur } \\
\text { - Combinaison de différents phénomènes électrochimiques et } \\
\text { - } \text { physicochimiques }\end{array}$ & $\begin{array}{l}\text { - Gestion des boues chargées en ion ferreux ou aluminium } \\
\text { - Efficacité limitée sur l'élimination du } \mathrm{NH}_{4} \text { et des petites } \\
\text { molécules }\end{array}$ \\
\hline
\end{tabular}

${ }^{\mathrm{a}} \mathrm{DCO}:$ demande chimique en oxygène

\subsection{Couplage électro-oxydation et traitement biologique}

Le couplage des techniques d'électro-oxydation et biologiques a été testé sur différents types de lixiviats avec des résultats satisfaisants. Les études existantes portent sur des lixiviats "jeunes " avec de fortes concentrations en DCO (QUAN et al., 2013) et des lixiviats " matures " qui présentent un rapport de biodégradabilité (DBO/DCO) faible (FEKI et al., 2009). Il est à noter que, dans ce couplage, le procédé d'électro-oxydation est généralement placé en aval du traitement biologique (ANGLADA et al., 2010; FEKI et al., 2009; FERNANDES et al., 2012; QUAN et al., 2013; WANG et al., 2001; XIAO et al., 2013). Ce choix peut être justifié du fait qu'il peut y avoir un phénomène de compétition entre la dégradation électrochimique des composés réfractaires et celle des composés biodégradables. Installée en aval du traitement biologique, l'électro-oxydation permettra une dégradation des matières résiduelles récalcitrantes. Néanmoins, d'autres auteurs ont opté pour une configuration différente en plaçant le procédé électrochimique en amont du procédé biologique dans le but de convertir les composés bioréfractaires en molécules facilement biodégradables (LI et al., 2007). Le tableau 7 résume quelques résultats obtenus après électro-oxydation et traitement biologique des lixiviats.

Après une étape de traitement dans un réacteur anaérobie (temps de rétention de 6,1 jours et une charge organique de $0,78 \mathrm{~g}$ DCO $\left.\cdot \mathrm{L}^{-1} \cdot \mathrm{j}^{-1}\right)$, le lixiviat obtenu par WANG et al. (2001) présentait des concentrations en $\mathrm{DCO}$ et en $\mathrm{NH}_{4}$ de $1610 \mathrm{mg} \cdot \mathrm{L}^{-1}$ et $1480 \mathrm{mg} \cdot \mathrm{L}^{-1}$ respectivement, avec un rapport de biodégradabilité (DBO/DCO) de 0,06. En soumettant ce lixiviat au traitement d'électro-oxydation pendant une période de $6 \mathrm{~h}$, la $\mathrm{DCO}$ et le $\mathrm{NH}_{4}$ ont été respectivement éliminés à hauteur de $87 \%$ et $100 \%$ dans les conditions suivantes : $i=32,3 \mathrm{~mA} \cdot \mathrm{cm}^{-2}$, concentration initiale de $\mathrm{Cl}^{-}=4000 \mathrm{mg} \cdot \mathrm{L}^{-1}$ et une anode en $\mathrm{Ti} / \mathrm{SnO}_{2}$. Les auteurs ont justifié ces taux d'élimination élevés de la DCO et de l'azote ammoniacal par l'apport additionnel d'ions chlorures dont l'oxydation induit la formation d'oxydants (acide hypochloreux, $\mathrm{HClO}$ ) qui contribuent à la dépollution (section 3. Procédé d'électrooxydation). Dans les mêmes conditions de traitement, les taux d'élimination de la DCO et du $\mathrm{NH}_{4}$ sont de $45,5 \%$ et 50,4\% respectivement sans ajout de chlorure. QUAN et al. (2013) et FEKI et al. (2009) ont rapporté également des résultats satisfaisants en termes de diminution de la DCO et de $\mathrm{NH}_{4}$ après $1 \mathrm{~h}$ de traitement avec une concentration initiale en chlorure de l'ordre de $5000 \mathrm{mg} \cdot \mathrm{L}^{-1}$ sur des lixiviats prétraités biologiquement.

FERNANDES et al. (2012) ont étudié les performances épuratoires de l'électro-oxydation d'un lixiviat préalablement traité par un procédé de boue activée. Le diamant dopé au bore (DDB) est utilisé comme électrode de travail. Le traitement biologique a permis de réduire la concentration de DCO à $5800 \mathrm{mg} \cdot \mathrm{L}^{-1}$ et celle de $\mathrm{NH}_{4}$ à $1210 \mathrm{mg} \cdot \mathrm{L}^{-1}$ avec des concentrations initiales respectives de $13700 \mathrm{mg} \cdot \mathrm{L}^{-1}$ et de $1880 \mathrm{mg} \cdot \mathrm{L}^{-1}$. Après $6 \mathrm{~h}$ de traitement d'électro-oxydation avec une densité de courant de $30 \mathrm{~mA} \cdot \mathrm{cm}^{-2}$, les concentrations résiduelles de $\mathrm{DCO}$ et de $\mathrm{NH}_{4}$ étaient respectivement $4600 \mathrm{mg} \cdot \mathrm{L}^{-1}$ et $1050 \mathrm{mg} \cdot \mathrm{L}^{-1}$. Ce qui représente des taux d'élimination de la DCO de $27 \%$ et de $14 \%$ pour le $\mathrm{NH}_{4}$. On constate que les concentrations obtenues après traitement électrochimique sont encore élevées. Ces résultats peuvent être expliqués par les concentrations élevées en DCO et en 
Tableau 7. Résultats obtenus après électro-oxydation et traitement biologique des lixiviats de site d'enfouissement.

Table $7 \quad$ Results obtained after landfill leachate treatment by electro-oxidation and biological processes.

\begin{tabular}{|c|c|c|c|c|c|c|c|}
\hline \multirow{2}{*}{$\begin{array}{l}\text { Ordre des } \\
\text { traitements }\end{array}$} & \multirow{2}{*}{$\begin{array}{l}\text { Traitement } \\
\text { biologique }\end{array}$} & \multirow{2}{*}{$\begin{array}{c}\text { Traitement } \\
\text { électrochimique }\end{array}$} & \multicolumn{2}{|c|}{$\begin{array}{c}\text { Performance du traitement } \\
\text { biologique }\end{array}$} & \multicolumn{2}{|c|}{$\begin{array}{c}\text { Performance du traitement } \\
\text { électrochimique }\end{array}$} & \multirow{2}{*}{ Références } \\
\hline & & & $\begin{array}{c}\text { Enlèvement } \\
\text { DCO }(\%)\end{array}$ & $\begin{array}{c}\text { Enlèvement } \\
\mathrm{NH}_{4}(\%)\end{array}$ & $\begin{array}{c}\text { Enlèvement } \\
\text { DCO }(\%)\end{array}$ & $\begin{array}{c}\text { Enlèvement } \\
\mathrm{NH}_{4}(\%)\end{array}$ & \\
\hline Bio $\rightarrow$ EO & $\begin{array}{l}\text { Système aérobie et anaérobie } \\
\text { en séquence }\end{array}$ & $\begin{array}{l}\text { Anode : Ti } / \mathrm{RuO}_{2}-\mathrm{IrO}_{2} \\
i=65 \mathrm{~mA} \cdot \mathrm{cm}^{-2} ; t=1 \mathrm{~h}\end{array}$ & 96 & 97 & 85 & 97 & QUAN et al., 2013 \\
\hline Bio $\rightarrow$ EO & 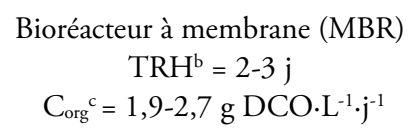 & $\begin{array}{c}\quad \text { Anode }: \mathrm{Ti} / \mathrm{Pt} \\
i=40 \mathrm{~mA} \cdot \mathrm{cm}^{-2} ; t=1 \mathrm{~h}\end{array}$ & 61 & 73 & 63 & 80 & FEKI et al., 2009 \\
\hline $\mathrm{EO} \rightarrow \mathrm{Bio}$ & $\begin{array}{c}\text { Réacteur anaérobie (UASB) } \\
\text { TRH }=16 \mathrm{~h} \\
\text { Corg }=8 \mathrm{~kg} \mathrm{DCO} \cdot \mathrm{m}^{-3} \cdot \mathrm{j}^{-1}\end{array}$ & $\begin{aligned} & \text { Anode }: \text { Ti-Ir-Ru } \\
i= & 150 \mathrm{~mA} \cdot \mathrm{cm}^{-2} ; t=1 \mathrm{~h}\end{aligned}$ & $69-91$ & $5-15,5$ & 53,5 & 77,5 & LI et al., 2007 \\
\hline Bio $\rightarrow \mathrm{CC} \rightarrow \mathrm{EO}$ & $\begin{array}{l}\text { Traitement aérobie } \\
\text { et anaérobie }\end{array}$ & $\begin{array}{c}\text { Anode }: \mathrm{RuO}_{2} / \mathrm{Ti} \\
i=50 \mathrm{~mA} \cdot \mathrm{cm}^{-2} ; \mathrm{t}=4 \mathrm{~h}\end{array}$ & - & - & 88 & 65 & XIAO et al., 2013 \\
\hline $\mathrm{Bio} \rightarrow \mathrm{EO}$ & $\begin{array}{c}\text { Réacteur anaérobie (UASB) } \\
\qquad \begin{array}{c}\text { TRH }=6,1 \mathrm{j} \\
\text { Corg }=0,78 \mathrm{~g} \mathrm{DCO} \cdot \mathrm{L}^{-1} \cdot \mathrm{j}^{-1}\end{array}\end{array}$ & $\begin{array}{c}\quad \text { Anode }: \mathrm{Ti} / \mathrm{SnO}_{2} \\
i=32,3 \mathrm{~mA} \cdot \mathrm{cm}^{-2} ; t=6 \mathrm{~h}\end{array}$ & 66 & 0 & 87 & 100 & WANG et al., 2001 \\
\hline Bio $\rightarrow$ EO & $\begin{array}{l}\text { Réacteur biologique séquentiel } \\
\qquad(\mathrm{SBR}) ; \mathrm{TRH}=12 \mathrm{~h}\end{array}$ & $\begin{array}{c}\text { Anode : } \mathrm{Ti} / \mathrm{TiO}_{2}-\mathrm{IrO}_{2} \\
i=0,8-4,8 \mathrm{~mA} \cdot \mathrm{cm}^{-2} ; t=3 \mathrm{~h}\end{array}$ & $>95$ & 78 & $30-70$ & $30-100$ & CHU et al., 2008 \\
\hline $\mathrm{Bio} \rightarrow \mathrm{EO}$ & $\begin{array}{l}\text { Réacteur biologique séquentiel } \\
\text { (SBR) }\end{array}$ & $\begin{array}{c}\text { Anode : BDD } \\
I=15-21 \mathrm{~A} ; t=4 \mathrm{~h}\end{array}$ & $>85$ & - & 90 & - & PAPASTAVROU et al., 2009 \\
\hline Bio $\rightarrow$ EO & $\begin{array}{c}\text { Bioréacteur à membrane (MBR) } \\
\text { TRH }=2-3 \mathrm{j} \\
\mathrm{C}_{\text {org }}=1,9-2,7 \mathrm{~g} \mathrm{DCO} \cdot \mathrm{L}^{-1} \cdot \mathrm{j}^{-1}\end{array}$ & $\begin{array}{c}\text { Anode }: \mathrm{PbO}_{2} \\
i=40 \mathrm{~mA} \cdot \mathrm{cm}^{-2} ; t=1 \mathrm{~h}\end{array}$ & 61 & 73 & 30 & 58 & FEKI et al., 2009 \\
\hline Bio $\rightarrow$ EO & Boue activée & $\begin{array}{c}\text { Anode : BDD } \\
i=50 \mathrm{~mA} \cdot \mathrm{cm}^{-2} ; t=6 \mathrm{~h}\end{array}$ & 57,6 & 35,6 & 27 & 14 & FERNANDES et al., 2012 \\
\hline
\end{tabular}

${ }^{\mathrm{a}}$ Bio : traitement biologique ; EO : électro-oxydation ; CC : coagulation chimique

${ }^{\mathrm{b}} \mathrm{THR}$ : temps de rétention hydraulique

${ }^{\mathrm{e}} \mathrm{C}_{\mathrm{org}}$ : charge organique ; DCO : demande chimique en oxygène 
$\mathrm{NH}_{4}\left(4600 \mathrm{mg} \cdot \mathrm{L}^{-1}\right.$ et $\left.1050 \mathrm{mg} \cdot \mathrm{L}^{-1}\right)$ à l'entrée du procédé électrochimique. Ceci montre qu'un traitement biologique poussé est souhaitable pour réduire les charges organiques à l'entrée du procédé électrochimique. Néanmoins, le procédé électrochimique a permis d'éliminer $1520 \mathrm{mg} \cdot \mathrm{L}^{-1}$ de DCO et $160 \mathrm{mg} \cdot \mathrm{L}^{-1}$ de $\mathrm{N}-\mathrm{NH}_{4}$. Des analyses spectrophotométriques effectuées sur l'effluent électro-oxydé ont révélé une diminution de $43 \%$ de l'absorbance à $275 \mathrm{~nm}$, mettant ainsi en évidence une réduction des composés aromatiques biorécalcitrants.

Suite à une séquence de traitement aérobie et anaérobie suivie d'une coagulation chimique, le lixiviat obtenu par XIAO et al. (2013) présentait un faible rapport de biodégradabilité $\left(\mathrm{DBO} / \mathrm{DCO}=0,09, \mathrm{DCO} \approx 560 \mathrm{mg} \cdot \mathrm{L}^{-1}\right.$ et $\left.\mathrm{NH}_{4} \approx 700 \mathrm{mg} \cdot \mathrm{L}^{-1}\right)$. En soumettant l'effluent à un traitement d'électro-oxydation d'une durée de $4 \mathrm{~h}$ avec une densité de courant de $50 \mathrm{~mA} \cdot \mathrm{cm}^{-2}$, la $\mathrm{DCO}$ a été réduite de $88 \%$ et le $\mathrm{NH}_{4}$ de $65 \%$ avec une anode en titane et oxyde de ruthénium $\left(\mathrm{RuO}_{2} / \mathrm{Ti}\right)$. Des analyses chromatographiques et spectrophotométriques ont été réalisées avant et après traitement électrochimique. Les résultats ont montré qu'après l'électro-oxydation, les substances humiques et certaines molécules aromatiques sont transformées en des molécules plus biodégradables tels que les acides oxalique, butyrique et phénylacétique.

LI et al. (2007) ont travaillé avec une configuration différente des précédentes en plaçant un réacteur biologique anaérobie après le traitement électrochimique. Le lixiviat brut avait les caractéristiques suivantes : $\mathrm{DCO}=11450 \mathrm{mg} \cdot \mathrm{L}^{-1}$ et $\mathrm{NH}_{4}=2761 \mathrm{mg} \cdot \mathrm{L}^{-1}$ et $\mathrm{DBO} / \mathrm{DCO}=0,24$. Le traitement électrochimique $\left(i=150 \mathrm{~mA} \cdot \mathrm{cm}^{-2}, t=1 \mathrm{~h}\right.$ et anode en $\mathrm{Ti}-\mathrm{Ir}-\mathrm{Ru}$ ) a permis de réduire la DCO à $5320 \mathrm{mg} \cdot \mathrm{L}^{-1}$ et l'azote ammoniacal à $620 \mathrm{mg} \cdot \mathrm{L}^{-1}$ et d'augmenter le rapport de biodégradabilité de 0,24 à 0,5 . De plus, le pourcentage des acides organiques à faible masse moléculaire a augmenté de 28 à $90 \%$ ainsi que le pourcentage des acides gras volatils de 0,68 à $16,8 \%$ après l'étape d'électro-oxydation. L'effluent obtenu est traité dans un réacteur batch anaérobie (UASB) avec un temps de rétention hydraulique de 16 h et une charge organique de $8 \mathrm{~kg} \mathrm{DCO} \cdot \mathrm{m}^{-3} \cdot \mathrm{j}^{-1}$. La concentration finale en DCO varie de 472 à $1649 \mathrm{mg} \cdot \mathrm{L}^{-1}$ tandis que l'abattement de l'azote ammoniacal est très faible $(\approx 10 \%)$, ceci est lié au choix des conditions anaérobies du réacteur biologique.

\subsection{Combinaison électro-Fenton et traitement biologique}

À l'heure actuelle, le nombre d'études effectuées sur le couplage des procédés d'électro-Fenton $(\mathrm{EF})$ et biologiques pour le traitement des lixiviats est rudimentaire. La majeure partie des études existantes relate l'efficacité du procédé conventionnel de Fenton couplé à un traitement biologique (LOPEZ et al., 2004; WANG et al., 2012a; XIE et al., 2010b; DI IACONI et al., 2006). Néanmoins, il y a eu un certain nombre d'auteurs qui se sont intéressés à l'application de la technique d'électro-Fenton couplée à un traitement biologique pour traiter les lixiviats (LIN et CHANG, 2000; WANG et al., 2012b; ZHANG et al., 2013). Le tableau 8 récapitule les résultats obtenus suite au couplage de divers procédés électrochimiques avec des procédés biologiques pour le traitement des lixiviats.

LIN et CHANG (2000) ont couplé l'EF à un réacteur discontinu biologique (SBR) pour traiter les lixiviats obtenus après une première étape de coagulation chimique. L'effluent à traiter présentait un faible rapport de biodégradabilité (DBO/ $\mathrm{DCO}=0,1)$ et une concentration en $\mathrm{DCO}$ de $950 \mathrm{mg} \cdot \mathrm{L}^{-1}$. Le cycle de traitement biologique a duré $12 \mathrm{~h}$. Avec les conditions d'EF suivantes : concentration de $\mathrm{H}_{2} \mathrm{O}_{2}=750 \mathrm{mg} \cdot \mathrm{L}^{-1}$, $t=30 \mathrm{~min}, \mathrm{pH}=4, I=2,5 \mathrm{~A}$ et une anode en fer, la valeur finale de la DCO était inférieure à $100 \mathrm{mg} \cdot \mathrm{L}^{-1}$. Un rapport de biodégradabilité (DBO/DCO) de 0,3 a été enregistré suite à l'application du procédé d'électro-Fenton. WANG et al. (2012b) ont utilisé une cathode en carbone PTFE (polytétrafluoroéthylène) et un apport externe d'O $\mathrm{O}_{2}$ pour générer de façon électrochimique le peroxyde d'hydrogène afin de traiter un lixiviat biologiquement prétraité. Le traitement biologique est composé d'une succession d'un traitement dans un réacteur anaérobie (UASB), d'un traitement anoxie-aérobie suivi d'un bioréacteur à membrane (MBR). Le concentrat obtenu après traitement biologique est caractérisé par un rapport de biodégradabilité presque nul $\left(\mathrm{DCO}=3886 \mathrm{mg} \cdot \mathrm{L}^{-1}\right.$ et $\left.\mathrm{DBO}=2,6 \mathrm{mg} \cdot \mathrm{L}^{-1}\right)$ et une concentration en carbone organique total (COT) de $1347 \mathrm{mg} \cdot \mathrm{L}^{-1}$. Le traitement d'EF de cet effluent a permis d'éliminer $82 \%$ du COT après $6 \mathrm{~h}$ de traitement avec une densité de courant de $30 \mathrm{~mA} \cdot \mathrm{cm}^{-2}$ et $10 \mathrm{mM}$ de $\mathrm{FeSO}_{4}$. ZHANG et al. (2013) ont également expérimenté la combinaison d'un procédé biologique avec de l'EF. Le lixiviat a d'abord été traité dans un réacteur à biofilm (SBBR) avec un temps de cycle de $24 \mathrm{~h}$ puis soumis à $60 \mathrm{~min}$ de traitement $\mathrm{EF}$ avec une intensité de $2 \mathrm{~A}$, un $\mathrm{pH} 3$ et un ratio $\mathrm{H}_{2} \mathrm{O}_{2} / \mathrm{Fe}^{2+}$ de 12:1. L'efficacité globale du procédé en termes d'abattement de la DCO est d'environ $80 \%$ avec une valeur initiale de $2495 \mathrm{mg} \cdot \mathrm{L}^{-1}$. L'analyse de la distribution des poids molaires (PM) par ultrafiltration révèle une augmentation significative des composés dont le $\mathrm{PM}<4 \mathrm{kDa}$ après l'étape d'EF. La diminution de l'indice UV254 qui renseigne sur la dégradation des substances humiques est observée à l'issu du traitement électrochimique.

Il est intéressant de noter que ce couplage est appliqué sur d'autres types d'effluents caractérisés par de fortes charges de DCO ou par la présence de composés réfractaires. Par exemple, BABU et al. (2009) ont rapporté une performance globale d'élimination de la DCO de $86 \%$ en couplant l'EF à un procédé anaérobie pour traiter un effluent d'huile de palme. FERRAG-SIAGH et al. (2013) ont utilisé la même approche pour la dégradation de la tétracycline, composé caractérisé 
Tableau 8. Résultats obtenus sur le couplage des procédés électrochimiques et biologiques pour traiter les lixiviats.

Table 8. Results obtained after combination of electrochemical and biological processes for landfill leachate treatment.

\begin{tabular}{|c|c|c|c|c|c|}
\hline $\begin{array}{l}\text { Ordre des } \\
\text { traitements }^{\mathrm{a}}\end{array}$ & $\begin{array}{l}\text { Traitement } \\
\text { biologique }\end{array}$ & $\begin{array}{l}\text { Conditions du traitement } \\
\text { électrochimique }\end{array}$ & $\begin{array}{c}\begin{array}{c}\text { Concentration avant } \\
\text { traitement électrochimique }\end{array} \\
\text { DCO }(\mathrm{COT})^{\mathrm{d}} \\
\left(\mathrm{mg} \cdot \mathrm{L}^{-1}\right)\end{array}$ & $\begin{array}{c}\begin{array}{c}\text { Performance du } \\
\text { traitement }\end{array} \\
\text { DCO ou COT } \\
\text { (\% enlèvement) }\end{array}$ & Références \\
\hline Bio $\rightarrow$ EF & $\begin{array}{l}\text { UASB - anaérobie/ } \\
\text { aérobie - } \mathrm{MBR}^{\mathrm{b}}\end{array}$ & 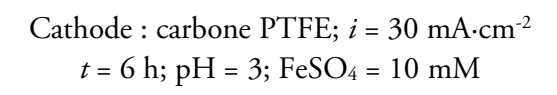 & $3886(1347)$ & $82 \mathrm{COT}$ & WANG et al., 2012b \\
\hline $\mathrm{CC} \rightarrow \mathrm{EF} \rightarrow$ Bio & $\begin{array}{l}\text { Réacteur biologique } \\
\text { séquentiel (SBR) }\end{array}$ & $\begin{array}{c}\text { Anode }: \mathrm{Fe} ; I=2,5 \mathrm{~A} ; t=30 \mathrm{~min} \\
\mathrm{pH}=4 ; \mathrm{CH}_{2} \mathrm{O} 2=750 \mathrm{mg} \cdot \mathrm{L}^{-1}\end{array}$ & 1914 & $67,3 \mathrm{DCO}$ & LIN et CHANG, 2000 \\
\hline Bio $\rightarrow$ EF & $\begin{array}{l}\text { Réacteur biologique } \\
\text { séquentiel (SBR) }\end{array}$ & $\begin{array}{c}I=2 \mathrm{~A} ; t=1 \mathrm{~h} \\
\mathrm{pH}=3 ; \mathrm{H}_{2} \mathrm{O}_{2} / \mathrm{Fe}^{2+}=12: 1\end{array}$ & 2046 & $71 \mathrm{DCO}$ & ZHANG et al., 2013 \\
\hline $\begin{array}{l}\text { Bio } \rightarrow \text { UF/NF } \rightarrow \\
\text { EC du concentrat }\end{array}$ & $\begin{array}{l}\text { Nitrification/ } \\
\text { dénitrification }\end{array}$ & $\begin{array}{c}\text { Anode }: \mathrm{Al} \\
i=15,9 \mathrm{~mA} \cdot \mathrm{cm}^{-2} ; t=30 \mathrm{~min}\end{array}$ & - & $45 \mathrm{DCO}$ & TOP et al., 2011 \\
\hline $\mathrm{Bio} \rightarrow \mathrm{EC}+\mathrm{EO}$ & Traitement biologique & $\begin{array}{c}\text { (EC) anode : Fe; } V=4 \mathrm{~V} ; t=1 \mathrm{~h} \\
\text { (EO) cathode }: \mathrm{DDB}^{\mathrm{c}} \\
i=30 \mathrm{~mA} \cdot \mathrm{cm}^{-2} ; t=8 \mathrm{~h}\end{array}$ & 11720 & $81 \mathrm{DCO}$ & NORMA et al., 2012a \\
\hline $\mathrm{Bio}+\mathrm{CC} \rightarrow \mathrm{PEC}$ & $\begin{array}{l}\text { Traitements aérobie } \\
\text { et anaérobie }\end{array}$ & $\begin{array}{c}\text { Anode : } \mathrm{RuO}_{2} / \mathrm{Ti} ; i=50 \mathrm{~mA} \cdot \mathrm{cm}^{-2} \\
\quad t=4 \mathrm{~h} ; \mathrm{UV}(20 \mathrm{~W} ; 254 \mathrm{~nm})\end{array}$ & 560 & $91 \mathrm{DCO}$ & XIAO et al., 2013 \\
\hline $\mathrm{Bio} \rightarrow \mathrm{PEC}$ & $\begin{array}{l}\text { Traitement } \\
\text { biologique }\end{array}$ & $\begin{array}{c}\text { Anode }: \mathrm{Ti} / \mathrm{TiRuO}_{2} \\
i=67,1 \mathrm{~mA} \cdot \mathrm{cm}^{-2} ; t=2,5 \mathrm{~h} \\
\mathrm{UV}\left(10 \mathrm{~W} ; 253,7 \mathrm{~nm} ; 1,36 \mathrm{~mW} \cdot \mathrm{cm}^{-2}\right)\end{array}$ & 560 & 74,1 DCO & ZHAO et al., 2010 \\
\hline $\begin{array}{l}\text { Bio } \rightarrow \text { NF/OI } \rightarrow \\
\text { EP du concentrat }\end{array}$ & $\begin{array}{l}\text { UASB - anaérobie/ } \\
\text { aérobie - MBR }\end{array}$ & $\begin{array}{c}\text { Cathode : carbone PTFE } ; i=17,5 \mathrm{~mA} \cdot \mathrm{cm}^{-2} \\
t=4 \mathrm{~min} ; \text { débit }\left(\mathrm{O}_{2+} \mathrm{O}_{3}\right)=0,3 \mathrm{~L} \cdot \mathrm{min}^{-1} \\
\mathrm{C}_{\mathrm{H} 2 \mathrm{O} 2}=157 \mathrm{mg} \cdot \mathrm{L}^{-1}\end{array}$ & $(1650)$ & $87 \mathrm{COT}$ & LI et al., 2013 \\
\hline
\end{tabular}

${ }^{a}$ Bio : biologique; EF : électro-Fenton; CC : coagulation chimique; UF : ultrafiltration; NF : nanofiltration; EC : électrocoagulation; EO : électro-oxydation; PEC : photo-

électrochimique; OI : osmose inverse; EP : électro-peroxone

bUASB : réacteur anaérobie; $\mathrm{MBR}$ : bioréacteur à membrane

${ }^{c}$ DDB : diamant dopé au bore

${ }^{\mathrm{d}} \mathrm{DCO}$ : demande chimique en oxygène ; COT : carbone organique total 
par des noyaux aromatiques difficilement biodégradables. Le couplage du procédé classique de Fenton à une filière biologique s'est également montré efficace pour le traitement des lixiviats (LOPEZ et al., 2004; WANG et al., 2012a). XIE et al. (2010b) ont développé un pilote d'une capacité de $20 \mathrm{~m}^{3} \cdot \mathrm{j}^{-1}$ pour traiter un lixiviat mature en plaçant le procédé de Fenton classique entre deux filtres biologiques. Les valeurs de la DCO et du $\mathrm{NH}_{4}$ après traitement sont inférieures à 100 et $25 \mathrm{mg} \cdot \mathrm{L}^{-1}$, respectivement.

\subsection{Combinaison électrocoagulation et traitement biologique}

Tout comme l'EF, il existe très peu d'études effectuées sur le couplage des procédés d'électrocoagulation et biologiques pour traiter les lixiviats. NORMA et al. (2012a) ont expérimenté les procédés d'EC et d'EO sur un lixiviat obtenu après une étape de traitement biologique. Suite au traitement biologique, la valeur de la DCO était de $11720 \mathrm{mg} \cdot \mathrm{L}^{-1}$. Le fer et le diamant dopé au bore (DDB) ont été utilisés comme anodes pour l'EC et l'EO, respectivement. L'acier inoxydable a été employé comme cathode dans les deux modes de traitement. Leffluent est d'abord électrocoagulé pendant une heure avec une tension de $4 \mathrm{~V}$ sans agitation. Après une étape intermédiaire de filtration, le perméat obtenu est soumis à $8 \mathrm{~h}$ d'électro-oxydation avec une densité de courant de $30 \mathrm{~mA} \cdot \mathrm{cm}^{-2}$. Le traitement successif d'EC et d'EO a permis la réduction de $81 \%$ de la DCO et de $50 \%$ du COT.

TOP et al. (2011) ont aussi étudié l'EC d'un concentrat obtenu après une étape de traitement biologique et de filtration. Le lixiviat est traité dans un premier temps par un procédé de nitrification/dénitrification. L'effluent est ensuite filtré dans deux modules d'ultrafiltration et de nanofiltration. Le concentrat obtenu contenait une large partie des grosses molécules réfractaires non dégradées par la filière biologique et retenues par les filtres. Pour traiter le concentrat, les auteurs ont utilisé quatre plaques d'aluminium (deux anodes et deux cathodes) d'une surface totale de $252 \mathrm{~cm}^{2}$ en configuration monopolaire. Avec une densité de courant de 15,9 $\mathrm{mA} \cdot \mathrm{cm}^{-2}$ et un temps de traitement de $30 \mathrm{~min}, 45 \%$ de la DCO est éliminée et $60 \%$ de la couleur. La concentration initiale de DCO était de $6200 \mathrm{mg} \cdot \mathrm{L}^{-1}$.

\subsection{Couplage des autres procédés électrochimiques avec un} traitement biologique

D’autres procédés électrochimiques sont également utilisés en combinaison avec les procédés biologiques pour le traitement des lixiviats. On peut citer le procédé photo-électrochimique et le procédé d'électro-peroxone. Le procédé photo-électrochimique a le même principe que le procédé d'électro-oxydation (section 3. Procédé d'électro-oxydation) avec l'ajout d'une source de lumière ultraviolette. Les radiations UV peuvent avoir un effet destructif sur les molécules organiques et favoriser la formation de radicaux puissants comme $\mathrm{OH}^{\bullet}$ et $\mathrm{HClO}$ dans la solution (TAUCHERT et al., 2006). Le procédé d'électro-peroxone (EP) est dérivé du procédé Peroxone qui consiste à mélanger l'ozone et le peroxyde d'hydrogène afin de former des radicaux hydroxyles (Équation 23).

$$
\mathrm{H}_{2} \mathrm{O}_{2}+2 \mathrm{O}_{3} \rightarrow 2 \mathrm{OH}^{\bullet}+3 \mathrm{O}_{2}
$$

Dans le procédé d'EP, le peroxyde d'hydrogène est généré de façon électrochimique à la cathode. L'ozone est introduit dans le réacteur après une étape d'ozonation à partir du dioxygène (LI et al., 2013). ZHAO et al. (2010) ont étudié l'influence du procédé photo-électrochimique (PEC) sur un lixiviat ayant subi un prétraitement biologique. Après traitement biologique, l'effluent présentait des concentrations en DCO de $560 \mathrm{mg} \cdot \mathrm{L}^{-1}$, en $\mathrm{NH}_{4}$ de $700 \mathrm{mg} \cdot \mathrm{L}^{-1}$ et un rapport de biodégradabilité faible $(\mathrm{DBO} / \mathrm{DCO}=0,07)$. Létude a été réalisée sur un pilote d'une capacité de 6,5 L. L'anode en DSA $\left(\mathrm{Ti} / \mathrm{TiRuO}_{2}\right)$ est constituée d'un tube concentrique tandis que la paroi extérieure du réacteur en acier inoxydable fait office de cathode. La source de radiation est assurée par une lampe UV, d'une puissance de $20 \mathrm{~W}$ et d'une longueur d'onde de $254 \mathrm{~nm}$, placée au milieu du réacteur. Dans les conditions optimales de traitement $\left(i=67,1 \mathrm{~mA} \cdot \mathrm{cm}^{-2}\right.$ et $t=2,5 \mathrm{~h}$ ), les taux d'abattement sont de $74,1 \%$ pour la DCO, de 41,6 \% pour le COT et de 94,5\% pour le $\mathrm{NH}_{4}$. Le rapport de biodégradabilité a aussi été augmenté de façon significative de 0,07 à 0,6 après le traitement PEC. XIAO et al. (2013) ont réalisé la même étude sur un lixiviat prétraité par un procédé aérobie/anaérobie et une coagulation chimique. Après l'étape de prétraitement, la $\mathrm{DCO}$ et le $\mathrm{NH}_{4}$ ont des valeurs moyennes respectives de 560 et $700 \mathrm{mg} \cdot \mathrm{L}^{-1}$. Dans le système utilisé, l'anode est constituée de titane et d'oxyde de ruthénium $\left(\mathrm{RuO}_{2} / \mathrm{Ti}\right)$ et la cathode de titane. Une lampe de mercure à basse pression ( $10 \mathrm{~W}$ et $253,7 \mathrm{~nm}$ ) est utilisée comme source de radiation UV. Un taux d'abattement de $91 \%$ de la DCO et une élimination quasi complète de la couleur et de l'azote ammoniacal ont été enregistrés avec une densité de courant de $50 \mathrm{~mA} \cdot \mathrm{cm}^{-2}$ et un temps de traitement de $4 \mathrm{~h}$. Des expériences parallèles effectuées lors des deux études précédentes montrent une meilleure efficacité du procédé PEC par rapport au procédé d'électro-oxydation en termes d'élimination de la DCO, du COT et du $\mathrm{NH}_{4}$. Ceci peut s'expliquer par la photo-génération des radicaux hydroxyles et hypochloreux.

LI et al. (2013) ont étudié le procédé d'électro-peroxone sur un concentrat obtenu à la suite d'un traitement biologique et membranaire d'un lixiviat. Le traitement biologique est réalisé avec une succession d'un réacteur anaérobie (UASB), d'un traitement anoxie-aérobie suivi d'un bioréacteur à membrane (MBR). Le traitement membranaire est effectué sur deux modules de nanofiltration et d'osmose inverse. Le concentrat issu des procédés membranaires est principalement constitué 
de composés organiques réfractaires avec une concentration en COT de $1650 \mathrm{mg} \cdot \mathrm{L}^{-1}$. Un mélange d'ozone et de dioxygène est injecté dans le réacteur avec un débit de $0,3 \mathrm{~L} \cdot \mathrm{min}^{-1}$. Le peroxyde d'hydrogène est généré de façon électrochimique à la cathode en carbone PTFE (polytétrafluoroéthylène) à partir de l'oxygène dissous. Avec une densité de courant de $17,5 \mathrm{~mA} \cdot \mathrm{cm}^{-2}$ et un temps de traitement de $4 \mathrm{~h}$, le COT est éliminé à $87 \%$.

\section{TENDANCES FUTURES ET PERSPEC- TIVES DES PROCÉDÉS ÉLECTRO- CHIMIQUES ET LEUR COUPLAGE AVEC LES PROCÉDÉS BIOLOGIQUES}

Bien que l'utilisation des procédés électrochimiques pour le traitement des lixiviats ne soit pas encore très répandue, on constate depuis quelques années de plus en plus d'études réalisées sur ce sujet. Selon la base de données Scopus (Elsevier), 70 études ont été réalisées entre 2006 et 2013, sur l'utilisation des procédés d'EO, d'EF et d'EC pour le traitement des lixiviats par rapport à cinq études effectuées entre 1994 et 2005. L'utilisation modeste, mais croissante de ces techniques d'épuration est favorisée en partie par le vieillissement des décharges existantes qui produisent des lixiviats caractérisés par une pollution bioréfractaire et les normes de rejets qui deviennent de plus en plus contraignantes (MINISTÈRE QUÉBECOISE DU DÉVELOPPEMENT DURABLE DE L'ENVIRONNEMENT ET DES PARCS, 2005). En guise de comparaison, les normes de rejets concernant les lixiviats des LET sont presque similaires à celles des eaux usées urbaines. Or, ces dernières peuvent, dans certains cas, être jusqu'à 10 fois moins polluantes. Les procédés biologiques conventionnels se heurtent à la difficulté de traiter de façon convenable les lixiviats matures qui renferment un grand nombre de composés récalcitrants. Les effets directs et indirects du courant électrique permettent une dégradation partielle ou totale de ces polluants récalcitrants ou de les transférer dans une phase insoluble lors de l'EC. Les principaux atouts liés à l'utilisation des procédés électrochimiques sont : faible emprise au sol, temps de traitement court, automatisation facile, utilisation limitée de réactifs, bonne performance épuratoire et coût raisonnable. Malgré les intérêts qu'ils présentent, le potentiel des technologies électrochimiques reste encore sous-exploité dans le domaine du traitement des lixiviats. Selon la base de données Scopus, 130 études ont été réalisées depuis l'an 2000, sur l'utilisation du procédé classique de Fenton contre 17 études sur l'utilisation du procédé d'électro-Fenton pour le traitement des lixiviats. Sur cette même période de temps, on note 56 études sur la coagulation chimique contre 8 sur l'électrocoagulation.
Le procédé d'électro-oxydation permet à la fois d'éliminer la DCO et l'azote ammoniacal tout en augmentant la biodégradabilité de l'effluent. Cependant, les performances de l'EO sont beaucoup influencées par les ions responsables de l'oxydation indirecte. On peut amener à ajouter du chlorure de sodium pour activer l'oxydation indirecte dans le cas $\mathrm{du}$ traitement des lixiviats où les teneurs en $\mathrm{Cl}^{-}$sont faibles (<3 $\left.000 \mathrm{mg} \cdot \mathrm{L}^{-1}\right)$ (CHEN, 2004). Le potentiel toxique des sous-produits organiques chlorés formés après l'EO n'est pas encore explicitement étudié dans le cas des lixiviats. Une étude effectuée par NAUMCZYK et al. (1996) sur un effluent issu de l'industrie du textile chargé en chlorures révèle la formation de plusieurs sous-produits organochlorés après traitement d'EO. Parmi les sous-produits décelés par les auteurs on peut citer : le 1,1-dichlorocyclopentene, le 2,3-dichloro-2-méthylbutane, le chloromelhylsilane, etc. Avec l'avancement de la science des matériaux, l'arrivée d'autres électrodes plus performantes peut rendre la technique d'EO plus compétitive. Actuellement, l'électrode de diamant dopé au bore (DDB) se distingue des autres avec des résultats très satisfaisants. Les procédés d'EO peuvent être assistés par un procédé photochimique afin d'augmenter leur efficacité d'épuration. Par exemple, ZHAO et al. (2010) ont observé une augmentation de $27 \%$ de l'abattement de la DCO en couplant l'EO avec une source de radiation UV. En réalisant le même couplage, XIAO et al. (2013) ont obtenu une hausse de $27 \%$ et de $33 \%$ sur la cinétique de dégradation respective de la $\mathrm{DCO}$ et du $\mathrm{NH}_{4}$.

Le procédé d'EF est performant pour le traitement de la pollution organique avec l'implication du radical hydroxyle, qui est un oxydant puissant et non sélectif. Sa capacité à augmenter le rapport de biodégradabilité de l'effluent rend son couplage avec une filière de traitement biologique intéressant. Cependant, son inefficacité à éliminer le $\mathrm{NH}_{4}$ constitue son principal défaut. La condition de traitement en milieu acide $(\mathrm{pH}<4)$ peut également enfreindre l'utilisation de ce procédé quand il s'agit de traiter de grand volume d'effluent. Comparé au procédé de Fenton conventionnel, le traitement d'EF des lixiviats n'est pas encore suffisamment étudié dans tous ses aspects. Lors d'une opération de Fenton classique, les deux réactifs $\left(\mathrm{H}_{2} \mathrm{O}_{2}\right.$ et $\left.\mathrm{Fe}^{2+}\right)$ sont introduits dans le réacteur avec les proportions souhaitées. En revanche, il est délicat de respecter un ratio molaire $\mathrm{H}_{2} \mathrm{O}_{2} / \mathrm{Fe}^{2+}$ donné quand l'un ou les deux réactifs sont générés de façon électrochimique. Une divergence existe aussi sur le ratio optimal de $\mathrm{H}_{2} \mathrm{O}_{2} / \mathrm{Fe}^{2+}$ à appliquer lors du traitement des lixiviats. Le ratio $\mathrm{H}_{2} \mathrm{O}_{2} / \mathrm{Fe}^{2+}$ varie entre 1 et 12 dans les études consultées. Ceci est lié en partie à la diversité de compositions physico-chimiques des lixiviats et les différentes méthodes utilisées pour la détermination de ce ratio. L'optimisation du ratio et des quantités de $\mathrm{H}_{2} \mathrm{O}_{2}$ et $\mathrm{Fe}^{2+}$ générées permettra de réduire les boues obtenues lors des étapes de neutralisation en fin de traitement. Le couplage du procédé d'EF avec les techniques photochimiques : l'électro-photoFenton peut augmenter les performances épuratoires de l'EF 
mais s'accompagnera d'un surcoût. ZAZOULI et al. (2012) et KRZYSZTOSZEK et NAUMCZYK (2012) ont réalisé des études sur le procédé classique de Fenton couplé avec une radiation UV (photo-Fenton) pour le traitement des lixiviats. Leurs résultats ont montré une augmentation de l'abattement de la DCO (13,6\% et 22,4\% respectivement) avec le procédé photo-Fenton comparé à l'utilisation du Fenton seul. À l'état actuel de nos connaissances, aucune étude n'est encore faite sur le traitement des lixiviats avec l'utilisation du procédé d'électrophoto-Fenton.

Le procédé d'électrocoagulation est modestement étudié en traitement des lixiviats comparé au procédé traditionnel de coagulation chimique. Bien que des études récentes ont montré les avantages de l'EC en termes de quantité de boues produites et d'élimination de la DCO (ILHAN et al., 2008; MARIAM et NGHIEM, 2010), la coagulation chimique est encore largement utilisée. L'EC présente l'avantage de combiner à la fois les phénomènes physico-chimiques (coagulation, précipitation, complexation) et électrochimiques (électroflottation, oxydation directe et indirecte). Elle peut être opérée dans une large plage de $\mathrm{pH}$. Son efficacité à éliminer les grosses molécules et la turbidité fait qu'il est parfois utilisé comme étape de prétraitement ou de traitement tertiaire. Cependant, les limitations des transferts de masse et la gestion des résidus d'hydroxydes métalliques formés peuvent expliquer la sousutilisation de l'EC. Les études de laboratoire se font généralement avec des cellules électrolytiques de formes parallélépipédiques. La conception et l'expérimentation d'autres types de réacteurs permettront d'optimiser l'hydrodynamique du milieu et de réduire la limitation des transferts de masse. L'effet des configurations monopolaire et bipolaire des électrodes pendant l'EC des lixiviats reste un sujet à étudier.

Le couplage des procédés électrochimiques et biologiques présente trois principaux avantages : l'augmentation des performances épuratoires, la réduction des coûts d'opération et la diminution des temps de traitement. Pour le traitement des lixiviats " matures ", caractérisés par une forte présence en composés réfractaires, les procédés électrochimiques permettront de dégrader une partie des composés réfractaires et d'augmenter la biodégradabilité de l'effluent. L'ordre de la disposition des ouvrages électrochimiques et biologiques dépend des besoins du traitement. De ce fait, on peut placer le procédé biologique en amont afin de traiter les composés biodégradables et de finir le traitement des composés récalcitrants par le procédé électrochimique. L'autre disposition consiste à placer le procédé électrochimique en amont pour augmenter la biodégradabilité de l'effluent et de finir avec un traitement biologique. Il est important de choisir un procédé biologique capable de traiter l'azote ammoniacal dans le cas du couplage avec certains procédés électrochimiques. C'est le cas des procédés d'EF, d'EC et d'EP qui présentent une faible efficacité par rapport à l'élimination de l'azote ammoniacal. L'effluent peut être carencé en nutriments dans le cas où le procédé biologique est placé en aval de la filière électrochimique. C'est le cas du phosphore dont la concentration est généralement faible dans les lixiviats matures. Sur 106 lixiviats matures étudiés, KJELDSEN et CHRISTOPHERSEN (2001) ont rapporté une valeur moyenne de $\mathrm{Pt}=2,7 \mathrm{mg} \cdot \mathrm{L}^{-1}$. Dans certains cas, on peut être amené à rajouter une source de phosphore afin de respecter l'équilibre nutritif des bactéries (IMAI et al., 1995). La majeure partie des études est réalisée sur des lixiviats prétraités par une filière biologique existante. Des études détaillées qui s'intéressent à la fois aux procédés biologiques et électrochimiques restent rares. En vue d'augmenter les performances épuratoires et de lever les incertitudes techniques qui planent sur ce couplage, des études plus détaillées devront être réalisées dans le futur. Lors de ses études, l'influence du traitement biologique sur la qualité de traitement électrochimique devrait être clarifiée et vice-versa. Des études d'optimisation des différents paramètres opératoires de chacun des deux procédés permettront d'augmenter l'efficacité du couplage tout en réduisant les coûts opératoires.

\section{CONCLUSION}

Au cours de cette revue de littérature, nous avons abordé les trois principaux procédés électrochimiques (l'électro-oxydation, l'électro-Fenton et l'électrocoagulation) appliqués au traitement des lixiviats. Leurs combinaisons avec les procédés biologiques sont également étudiées. Les trois procédés électrochimiques présentent un avantage commun qui est la faculté de traiter les composés réfractaires présents dans les lixiviats. Les mécanismes réactionnels qui rentrent en jeu pendant le traitement sont différents d'un procédé à un autre. Pendant l'EO, les réactions directes et indirectes qui impliquent le radical hydroxyle et d'autres oxydants intermédiaires sont responsables de la dépollution. Lors du traitement EF, le radical hydroxyle issu de la réaction entre le peroxyde d'hydrogène et l'ion ferreux est à l'origine de la dégradation des polluants organiques. Concernant l'EC, le mécanisme de dépollution est plus complexe, car différents phénomènes électrochimiques (électroflottation, oxydation directe et indirecte) et physico-chimiques (coagulation, précipitation, complexation) rentrent en jeu. D’une façon générale, les principaux paramètres opératoires qui influencent l'efficacité de ces procédés sont : la densité de courant, le temps de traitement, le type d'électrode utilisé, la distance interélectrodes et la conductivité du milieu. Toutefois, l'optimisation de certains paramètres est essentielle pour une meilleure performance du traitement électrochimique. C'est le cas du $\mathrm{pH}$ qui doit être compris entre 2 et 4,5 lors du procédé d'EF. 
Les procédés électrochimiques, utilisés dans les conditions optimales, donnent des résultats satisfaisants en termes d'élimination de la DCO (20 à $90 \%$ d'abattement selon le procédé utilisé et la concentration initiale), de la couleur et des germes pathogènes. L'utilisation de ces procédés pour le traitement des lixiviats " matures " permet d'éliminer les composés bioréfractaires et d'augmenter la biodégradabilité de l'effluent. Bien que l'application des technologiques électrochimiques pour le traitement des lixiviats soit en pleine expansion, plus d'études devront être faites pour l'optimisation de ces procédés. Les procédés d'électrocoagulation, d'électroFenton et d'électro-photo-Fenton sont modestement étudiés en comparaison des procédés traditionnels de coagulation chimique et de Fenton. Ceci est justifié par quelques désavantages que présentent ces techniques. La condition de traitement en milieu acide pendant l'EF peut être un frein lors du traitement de grand volume de lixiviat. La gestion des boues chargées en résidus métalliques est une contrainte qui doit être prise en compte lors de l'utilisation des procédés d'EF et d'EC. Les besoins énergétiques que requièrent ces procédés peuvent augmenter les coûts de traitement à l'échelle industrielle.

Le couplage des procédés électrochimiques et biologiques permet d'augmenter les performances épuratoires tout en réduisant les coûts et les temps de traitement. L'ordre de disposition des deux procédés dépend des besoins du traitement. Toutefois, des études approfondies sont nécessaires pour clarifier l'influence de chaque procédé sur l'autre procédé selon son placement dans la filière de traitement.

\section{REMERCIEMENTS}

Ce travail a été réalisé en collaboration avec le CRIQ (Centre de recherche industrielle du Québec) et les compagnies E2metrix et Gesterra. Il a également reçu le soutien financier du CRIBIQ (Consortium de recherche et innovations en bioprocédés industriels au Québec) et de MITACS.

\section{RÉFÉRENCES BIBLIOGRAPHIQUES}

ALOUI F., F. FKI, S. LOUKIL et S. SAYADI (2009). Application of combined membrane biological reactor and electro-oxidation processes for the treatment of landfill leachates. Water Sci. Technol., 60, 605-614.

ALTIN A. (2008). An alternative type of photoelectro-Fenton process for the treatment of landfill leachate. Sep. Purif. Technol., 61, 391-397.
AMOKRANE A., C. COMEL et J. VERON (1997). Landfill leachates pretreatment by coagulation-flocculation. Water Res., 31, 2775-2782.

ANGLADA A., A.M. URTIAGA et I. ORTIZ (2010). Laboratory and pilot plant scale study on the electrochemical oxidation of landfill leachate. J. Hazard. Mater., 181, 729735 .

ANGLADA A., A. URTIAGA, I. ORTIZ, D. MANTZAVINOS et E. DIAMADOPOULOS (2011). Boron-doped diamond anodic treatment of landfill leachate: evaluation of operating variables and formation of oxidation by-products. Water Res., 45, 828-838.

ASSELIN M. (2007). Utilisation de l'électrocoagulation dans le traitement des eaux usées industrielles chargées en matière organique. Mémoire de maîtrise, Univ. Québec, Institut national de la recherche scientifique, Canada, 92 p.

ATMACA E. (2009). Treatment of landfill leachate by using electro-Fenton method. J. Hazard. Mater., 163, 109-114.

AZIZ H.A., O.M. OTHMAN et S.S. ABU AMR (2013). The performance of electro-Fenton oxidation in the removal of coliform bacteria from landfill leachate. Waste Manage., 33, 396-400.

BABU B.R., K.S. MEERA, P. VENKATESAN et D. SUNANDHA (2009). Removal of fatty acids from palm oil effluent by combined electro-Fenton and biological oxidation process. Water, Air, Soil Poll., 211, 203-210.

BARLAZ M.A. et R.K. HAM (1993). Leachate and gas generation. Dans: Geotechnical practice for waste disposal. DANIEL D.E. (Éditeur), Chapman \& Hall, Londres, Royaume-Uni, pp. 113-136.

BASHIR M.J.K., M.H. ISA, S.R.M. KUTTY, Z.B. AWANG, H.A. AZIZ, S. MOHAJERI et I.H. FAROOQI (2009). Landfill leachate treatment by electrochemical oxidation. Waste Manage., 29, 2534-2541.

BAUN D.L. et T.H. CHRISTENSEN (2004). Speciation of heavy metals in landfill leachate: A review. Waste Manage. Res., 22, 3-23.

BODZEK M., E. ŁOBOS-MOYSA et M. ZAMOROWSKA (2006). Removal of organic compounds from municipal landfill leachate in a membrane bioreactor. Desalination, 198, 16-23. 
BOUCHER K., P. LESSARD, G. BUELNA et R. DUBÉ (2010). Biofiltration et captage des métaux lourds d'un lixiviat de lieu d'enfouissement de matières résiduelles. Salon des technologies environnementales du Québec, 16-17 mars, Québec, Canada.

BOUHEZILA F., M. HARITI, H. LOUNICI et N. MAMERI (2011). Treatment of the OUED SMAR town landfill leachate by an electrochemical reactor. Desalination, 280, 347-353.

BRILLAS E. et J. CASADO (2002). Aniline degradation by electro-Fenton and peroxi-coagulation processes using a flow reactor for wastewater treatment. Chemosphere, 47, 241-248.

BURTON S.A. et I.A. WATSON-CRAIK (1998). Ammonia and nitrogen fluxes in landfill sites: applicability to sustainable landfilling. Waste Manage. Res., 16, 41-53.

BUXTON G.V., C.L. GREENSTOCK, W.P. HELMAN et A.B. ROSS (1988). Critical review of rate constants for reactions of hydrated electrons, hydrogen atoms and hydroxyl radicals. J. Phys. Chem. Ref. Data, 17, 513-886.

CABEZA A., A. URTIAGA, M.J. RIVERO et I. ORTIZ (2007). Ammonium removal from landfill leachate by anodic oxidation. J. Hazard. Mater, 144, 715-719.

CALACE N., A. LIBERATORI, B.M. PETRONIO et M. PIETROLETTI (2001). Characteristics of different molecular weight fractions of organic matter in landfill leachate and their role in soil sorption of heavy metals. Environ. Pollut., 113, 331-339.

CANIZARES P., M. CARMONA, J.LOBATO, F. MARTINEZ et M. RODRIGO (2005a). Electrodissolution of aluminum electrodes in electrocoagulation processes. Ind. Eng. Chem. Res., 44, 4178-4185.

CANIZARES P., J. LOBATO, R. PAZ, M. RODRIGO et C. SÁEZ (2005b). Electrochemical oxidation of phenolic wastes with boron-doped diamond anodes. Water Res., 39, 2687-2703.

CHEN G. (2004). Electrochemical technologies in wastewater treatment. Sep. Purif. Technol., 38, 11-41.

CHEUNG K., L. CHU et M. WONG (1993). Toxic effect of landfill leachate on microalgae. Water, Air, Soil Poll., 69, 337-349.
CHIANG L.-C., J.-E. CHANG et T.-C. WEN (1995). Indirect oxidation effect in electrochemical oxidation treatment of landfill leachate. Water Res., 29, 671-678.

CHOU S., Y.-H. HUANG, S.-N. LEE, G.-H. HUANG et C. HUANG (1999). Treatment of high strength hexaminecontaining wastewater by electro-Fenton method. Water Res., 33, 751-759.

CHRISTENSEN J.B., D.L. JENSEN, C. GRØN, Z. FILIP et T.H. CHRISTENSEN (1998). Characterization of the dissolved organic carbon in landfill leachate-polluted groundwater. Water Res., 32, 125-135.

CHRISTENSEN T.H., P. KJELDSEN, H.J.R. ALBRECHTSEN, G. HERON, P.H. NIELSEN, P.L. BJERG et P.E. HOLM (1994). Attenuation of landfill leachate pollutants in aquifers. Crit. Rev. Env. Sci. Tec., 24, 119-202.

CHU Y., Q. ZHANG et D. XU (2008). Advanced treatment of landfill leachate from a sequencing batch reactor (SBR) by electrochemical oxidation process. J. Environ. Eng. Sci., 7, 627-633.

CLÉMENT B. et G. MERLIN (1995). The contribution of ammonia and alkalinity to landfill leachate toxicity to duckweed. Sci. Total Environ., 170, 71-79.

COMNINELLIS C. (1994). Electrocatalysis in the electrochemical conversion/combustion of organic pollutants for waste water treatment. Electrochim. Acta, 39, 1857-1862.

CONTRERAS J., M. VILLARROEL, R. NAVIA et M. TEUTLI (2009). Treating landfill leachate by electrocoagulation. Waste Manage. Res., 27, 534-541.

COSSU R., A.M. POLCARO, M.C. LAVAGNOLO, M. MASCIA, S. PALMAS et F. RENOLDI (1998). Electrochemical treatment of landfill leachate: oxidation at $\mathrm{Ti} / \mathrm{PbO}_{2}$ and $\mathrm{Ti} / \mathrm{SnO}_{2}$ anodes. Environ. Sci. Technol., 32, 3570-3573.

DENG Y. et J.D. ENGLEHARDT (2006). Treatment of landfill leachate by the Fenton process. Water Res., 40, 3683-3694.

DENG Y. et J.D. ENGLEHARDT (2007). Electrochemical oxidation for landfill leachate treatment. Waste Manage., 27, 380-388. 
DERMENTZIS K., A. CHRISTOFORIDIS et E. VALSAMIDOU (2011). Removal of nickel, copper, zinc and chromium from synthetic and industrial wastewater by electrocoagulation. Int. J. Environ. Sci., 1, 697-710.

DI IACONI C., R. RAMADORI et A. LOPEZ (2006). Combined biological and chemical degradation for treating a mature municipal landfill leachate. Biochem. Eng. J., 31, 118-124.

DROGUI P., S. ELMALEH, M. RUMEAU, C. BERNARD et A. RAMBAUD (2001). Hydrogen peroxide production by water electrolysis: Application to disinfection. J. Appl. Electrochem., 31, 877-882.

DROGUI P., J.-F. BLAIS et G. MERCIER (2007). Review of electrochemical technologies for environmental applications. Recent Pat. Eng., 1, 257-272.

DROGUI P., M. ASSELIN, S.K. BRAR, H. BENMOUSSA et J.-F. BLAIS (2008). Electrochemical removal of pollutants from agro-industry wastewaters. Sep. Purif. Technol., 61, 301-310.

EHRIG H.-J. (1983). Quality and quantity of sanitary landfill leachate. Waste Manage. Res., 1, 53-68.

EHRIG H.-J. (1989). Water and element balances of landfills. Dans: The landfill. BACCINI P. (Éditeur), Springer Berlin Heidelberg, Berlin, Allemagne, pp. 83-115.

FARMER J.C., F.T. WANG, R.A. HAWLEY-FEDDER, P.R. LEWIS, L.J. SUMMERS et L. FOILES (1992). Electrochemical treatment of mixed and hazardous wastes: oxidation of ethylene glycol and benzene by silver (II). J. Electrochem. Soc., 139, 654-662.

FEKI F., F. ALOUI, M. FEKI et S. SAYADI (2009). Electrochemical oxidation post-treatment of landfill leachates treated with membrane bioreactor. Chemosphere, $75,256-260$.

FENTON H. (1894). Oxidation of tartaric acid in presence of iron. J. Chem. Soc., 65, 899-910.

FERNANDES A., M.J. PACHECO, L. CIRÍACO et A. LOPES (2012). Anodic oxidation of a biologically treated leachate on a boron-doped diamond anode. $J$. Hazard. Mater., 199-200, 82-87.
FERNANDES A., P. SPRANGER, A.D. FONSECA, M.J. PACHECO, L. CIRÍACO et A. LOPES (2014). Effect of electrochemical treatments on the biodegradability of sanitary landfill leachates. Appl. Catal. B-Environ., 144, 514-520.

FERRAG-SIAGH F., F. FOURCADE, I. SOUTREL, H. AÏTAMAR, H. DJELAL et A. AMRANE (2013). Tetracycline degradation and mineralization by the coupling of an electro-Fenton pretreatment and a biological process. J. Chem. Technol. Biot., 88, 1380-1386.

FRASCARI D., F. BRONZINI, G. GIORDANO, G. TEDIOLI et M. NOCENTINI (2004). Long-term characterization, lagoon treatment and migration potential of landfill leachate: a case study in an active Italian landfill. Chemosphere, 54, 335-343.

GRIMM J., D. BESSARABOV et R. SANDERSON (1998). Review of electro-assisted methods for water purification. Desalination, 115, 285-294.

HARMSEN J. (1983). Identification of organic compounds in leachate from a waste tip. Water Res., 17, 699-705.

HENDERSON J.P. et J.W. ATWATER (1995). High ammonia landfill leachate treatment using anaerobic filter and rotating biological contactor. Can. J. Civil. Eng., 22, 992-1000.

HENRY J.G. et D. PRASAD (2000). Anaerobic treatment of landfill leachate by sulfate reduction. Water Sci. Technol., 41, 239-246.

HOILIJOKI T.H., R.H. KETTUNEN et J.A. RINTALA (2000). Nitrification of anaerobically pretreated municipal landfill leachate at low temperature. Water Res., 34, 14351446.

HOSOMI M., K. MATSUSIGE, Y. INAMORI, R. SUDO, K. YAMADA et Z. YOSHINO (1989). Sequencing batch reactor activated sludge processes for the treatment of municipal landfill leachate: Removal of nitrogen and refractory organic compounds. Water Sci. Technol., 21, 1651-1654.

ILHAN F., U. KURT, O. APAYDIN et M.T. GONULLU (2008). Treatment of leachate by electrocoagulation using aluminum and iron electrodes. J. Hazard. Mater., 154, 381-389. 
IM J.-H., H.-J. WOO, M.-W. CHOI, K.-B. HAN et C.-W. KIM (2001). Simultaneous organic and nitrogen removal from municipal landfill leachate using an anaerobic-aerobic system. Water Res., 35, 2403-2410.

IMAI A., K. ONUMA, Y. INAMORI et R. SUDO (1995). Biodegradation and adsorption in refractory leachate treatment by the biological activated carbon fluidized bed process. Water Res., 29, 687-694.

JENSEN D.L., A. LEDIN et T.H. CHRISTENSEN (1999). Speciation of heavy metals in landfill-leachate polluted groundwater. Water Res., 33, 2642-2650.

JOTIN R., S. IBRAHIM et N. HALIMOON (2012). Electro coagulation for removal of chemical oxygen demand in sanitary landfill leachate. Int. J. Environ. Sci., 3, 921-930

KABDAŞLI I., A. ŞAFAK et O. TÜNAY (2008). Bench-scale evaluation of treatment schemes incorporating struvite precipitation for young landfill leachate. Waste Manage., 28, 2386-2392.

KABUK H.A., F. İLHAN, Y. AVSAR, U. KURT, O. APAYDIN et M.T. GONULLU (2014). Investigation of Leachate Treatment with Electrocoagulation and Optimization by Response Surface Methodology. Clean - Soil, Air, Water, 42, 571-577.

KHANDEGARV.etA.K.SAROHA(2013).Electrocoagulation for the treatment of textile industry effluent - A review. J. Environ. Manage., 128, 949-963.

KIM S.M. et A. VOGELPOHL (1998). Degradation of organic pollutants by the photo-Fenton-process. Chem. Eng. Technol., 21, 187-191.

KJELDSEN P. et M. CHRISTOPHERSEN (2001). Composition of leachate from old landfills in Denmark. Waste Manage. Res., 19, 249-256.

KJELDSEN P., M.A. BARLAZ, A.P. ROOKER, A. BAUN, A. LEDIN et T.H. CHRISTENSEN (2002). Present and long-term composition of MSW landfill leachate: A review. Crit. Rev. Env. Sci. Tec., 32, 297-336.

KRZYSZTOSZEK A. et J. NAUMCZYK (2012). Landfill leachate treatment by Fenton, photo-Fenton processes and their modification. J. Adv. Oxid. Technol., 15, 53-63.

KURNIAWAN T.A., W. LO, G. CHAN et M.E. SILLANPAA (2010). Biological processes for treatment of landfill leachate. J. Environ. Monit., 12, 2032-2047.
LANZA M.R. et R. BERTAZZOLI (2002). Cyanide oxidation from wastewater in a flow electrochemical reactor. Ind. Eng. Chem. Res., 41, 22-26.

LEMA J., R. MENDEZ et R. BLAZQUEZ (1988). Characteristics of landfill leachates and alternatives for their treatment: a review. Water, Air, Soil Poll., 40, 223250 .

LI T., X. LI, J. CHEN, G. ZHANG et H. WANG (2007). Treatment of landfill leachate by electrochemical oxidation and anaerobic process. Water Environ. Res., 79, 514-520.

LI X., J. SONG, J. GUO, Z. WANG et Q. FENG (2011). Landfill leachate treatment using electrocoagulation. Procedia Environ. Sci., 10, 1159-1164.

LI X.-M., M. WANG, Z.-K. JIAO et Z. CHEN (2001). Study on electrolytic oxidation for landfill leachate treatment. China Water and Wastewater, 17, 14-17.

LI Z., S. YUAN, C. QIU, Y. WANG, X. PAN, J. WANG, C. WANG et J. ZUO (2013). Effective degradation of refractory organic pollutants in landfill leachate by electroperoxone treatment. Electrochim. Acta, 102, 174-182.

LIN S.H. et C.C. CHANG (2000). Treatment of landfill leachate by combined electro-Fenton oxidation and sequencing batch reactor method. Water Res., 34, 42434249.

LOPEZ A., M. PAGANO, A. VOLPE et A. CLAUDIO DI PINTO (2004). Fenton's pre-treatment of mature landfill leachate. Chemosphere, 54, 1005-1010.

MARIAM T. et L.D. NGHIEM (2010). Landfill leachate treatment using hybrid coagulation-nanofiltration processes. Desalination, 250, 677-681.

MARTIENSSEN M., R. SCHULZE et J. SIMON (1995). Capacities and limits of three different technologies for the biological treatment of leachate from solid waste landfill sites. Acta Biotechnol., 15, 269-276.

MEUNIER N., P. DROGUI, C. MONTANÉ, R. HAUSLER, J.-F. BLAIS et G. MERCIER (2006). Heavy metals removal from acidic and saline soil leachate using either electrochemical coagulation or chemical precipitation. J. Environ. Eng., 132, 545-554. 
MINISTÈRE QUÉBECOIS DU DÉVELOPPEMENT DURABLE DE L'ENVIRONNEMENT ET DES PARCS (2005). Règlement sur l'enfouissement et l'incinération de matières résiduelles. http://www2. publicationsduquebec.gouv.qc.ca/dynamicSearch/ telecharge.php?type =3\&file=/Q_2/Q2R19.HTM (consultation le 11 août 2014).

MOHAJERI S., H.A. AZIZ, M.H. ISA, M.A. ZAHED et M.N. ADLAN (2010). Statistical optimization of process parameters for landfill leachate treatment using electroFenton technique. J. Hazard. Mater., 176, 749-758.

MOLLAH M.Y.A., R. SCHENNACH, J.R. PARGA et D.L. COCKE (2001). Electrocoagulation (EC) - science and applications. J. Hazard. Mater., 84, 29-41.

MOLLAH M.Y.A., P. MORKOVSKY, J.A. GOMES, M. KESMEZ, J. PARGA et D.L. COCKE (2004). Fundamentals, present and future perspectives of electrocoagulation. J. Hazard. Mater., 114, 199-210.

MORAES P.B. et R. BERTAZZOLI (2005). Electrodegradation of landfill leachate in a flow electrochemical reactor. Chemosphere, 58, 41-46.

MORENO-CASILLAS H.A., D.L. COCKE, J.A.G. GOMES, P. MORKOVSKY, J.R. PARGA et E. PETERSON (2007). Electrocoagulation mechanism for COD removal. Sep. Purif. Technol., 56, 204-211.

NAUMCZYK J., L. SZPYRKOWICZ et F. ZILIO-GRANDI (1996). Electrochemical treatment of textile wastewater. Water Sci. Technol., 34, 17-24.

NIDHEESH P.V. et R. GANDHIMATHI (2012). Trends in electro-Fenton process for water and wastewater treatment: An overview. Desalination, 299, 1-15.

NORMA D., A. FERNANDES, L. CIRIACO, M.J. PACHECO et A. LOPES (2012a). Electrocoagulation and anodic oxidation as a complement of biological treatment of sanitary landfill leachates. Port. Electrochim. Acta, 30, 281-294.

NORMA D., A. FERNANDES, M.J. PACHECO, L. CIRIACO et A. LOPES (2012b). Electrocoagulation and anodic oxidation integrated process to treat leachate from a Portuguese sanitary landfill. Port. Electrochim. Acta, $30,221-234$.
ORKUN M.O. et A. KULEYIN (2012). Treatment performance evaluation of chemical oxygen demand from landfill leachate by electro-coagulation and electro-fenton technique. Environ. Prog. Sustain. Energy, 31, 59-67.

OSHODE O., A. BAKARE, A. ADEOGUN, M. EFUNTOYE et A. SOWUNMI (2008). Ecotoxicological assessment using Clarias gariepinus and microbial characterization of leachate from municipal solid waste landfill. Int. J. Environ. Res., 2, 391-400.

OYGARD J.K., A. MAGE et E. GJENGEDAL (2004). Estimation of the mass-balance of selected metals in four sanitary landfills in Western Norway, with emphasis on the heavy metal content of the deposited waste and the leachate. Water Res., 38, 2851-2858.

PAPASTAVROU C., D. MANTZAVINOS et E. DIAMADOPOULOS (2009). A comparative treatment of stabilized landfill leachate: coagulation and activated carbon adsorption vs. electrochemical oxidation. Environ. Technol., 30, 1547-1553.

PLOTKIN S. et N.M. RAM (1984). Multiple bioassays to assess the toxicity of a sanitary landfill leachate. Arch. Environ. Contam. Toxicol., 13, 197-206.

QIANG Z., J.-H. CHANG et C.-P. HUANG (2003). Electrochemical regeneration of $\mathrm{Fe}^{2+}$ in Fenton oxidation processes. Water Res., 37, 1308-1319.

QUAN X., Z. CHENG, B. CHEN et X. ZHU (2013). Electrochemical oxidation of recalcitrant organic compounds in biologically treated municipal solid waste leachate in a flow reactor. J. Environ. Sci., 25, 2023-2030.

RADA E., I. ISTRATE, M. RAGAZZI, G. ANDREOTTOLA et V. TORRETTA (2013). Analysis of electro-oxidation suitability for landfill leachate treatment through an experimental study. Sustainability, 5, 3960-3975.

RENOU S., J.G. GIVAUDAN, S. POULAIN, F. IRASSOUYAN et P. MOULIN (2008). Landfill leachate treatment: Review and opportunity. J. Hazard. Mater., 150, 468-493.

ROBINSON H.D. et G. GRANTHAM (1988). The treatment of landfill leachates in on-site aerated lagoon plants: experience in Britain and Ireland. Water Res., 22, 733-747. 
RULE J.H. (1979). Municipal landfill leachate in the ground and surface water, Chesapeake, Virginia: heavy metals. J. Environ. Health, 42, 60-64.

SERRANO K., P. MICHAUD, C. COMNINELLIS et A. SAVALL (2002). Electrochemical preparation of peroxodisulfuric acid using boron doped diamond thin film electrodes. Electrochim. Acta, 48, 431-436.

SINGH S.K., T.G. TOWNSEND et T.H. BOYER (2012). Evaluation of coagulation $\left(\mathrm{FeCl}_{3}\right)$ and anion exchange (MIEX) for stabilized landfill leachate treatment and highpressure membrane pretreatment. Sep. Purif. Technol., 96, 98-106.

STUCKI S., H. BAUMANN, H. CHRISTEN et R. KÖTZ (1987). Performance of a pressurized electrochemical ozone generator. J. Appl. Electrochem., 17, 773-778.

TANG W. et C. HUANG (1996). 2,4-Dichlorophenol oxidation kinetics by Fenton's reagent. Environ. Technol., 17, 1371-1378.

TATSI A.A., A.I. ZOUBOULIS, K.A. MATIS et P. SAMARAS (2003). Coagulation-flocculation pretreatment of sanitary landfill leachates. Chemosphere, 53, 737-744.

TAUCHERT E., S. SCHNEIDER, J.L. DE MORAIS et P. PERALTA-ZAMORA (2006). Photochemicallyassisted electrochemical degradation of landfill leachate. Chemosphere, 64, 1458-1463.

TOP S., E. SEKMAN, S. HOŞVER et M.S. BILGILI (2011). Characterization and electrocaogulative treatment of nanofiltration concentrate of a full-scale landfill leachate treatment plant. Desalination, 268, 158-162.

TRABELSI S., N. OTURAN, N. BELLAKHAL et M.A. OTURAN (2012). Application of Doehlert matrix to determine the optimal conditions for landfill leachate treatment by electro-Fenton process. J. Mater. Environ. Sci., 3, 426-433.

TSAI C.T., S.T. LIN, Y.C. SHUE et P.L. SU (1997). Electrolysis of soluble organic matter in leachate from landfills. Water Res., 31, 3073-3081.

UMAR M., H.A. AZIZ et M.S. YUSOFF (2010). Trends in the use of Fenton, electro-Fenton and photo-Fenton for the treatment of landfill leachate. Waste Manage., 30, 2113-2121.
VADILLO I., F. CARRASCO, B. ANDREO, A.G. DE TORRES et C. BOSCH (1999). Chemical composition of landfill leachate in a karst area with a Mediterranean climate (Marbella, southern Spain). Environ. Geol., 37, 326-332.

VLYSSIDES A.G., P.K. KARLIS et G. MAHNKEN (2003). Influence of various parameters on the electrochemical treatment of landfill leachates. J. Appl. Electrochem., 33, 155-159.

WALLING C. et A. GOOSEN (1973). Mechanism of the ferric ion catalyzed decomposition of hydrogen peroxide. Effect of organic substrates. J. Am. Chem. Soc., 95, 29872991.

WANG C.-T., W.-L. CHOU et Y.-M. KUO (2009a). Removal of COD from laundry wastewater by electrocoagulation/ electroflotation. J. Hazard. Mater., 164, 81-86.

WANG C.-T., W.-L. CHOU, M.-H. CHUNG et Y.-M. KUO (2010). COD removal from real dyeing wastewater by electro-Fenton technology using an activated carbon fiber cathode. Desalination, 253, 129-134.

WANG P., I.W. LAU et H.H. FANG (2001). Electrochemical oxidation of leachate pretreated in an upflow anaerobic sludge blanket reactor. Environ. Technol., 22, 373-381.

WANG X., S. CHEN, X. GU et K. WANG (2009b). Pilot study on the advanced treatment of landfill leachate using a combined coagulation, fenton oxidation and biological aerated filter process. Waste Manage., 29, 1354-1358.

WANG X., J. HAN, Z. CHEN, L. JIAN, X. GU et C.J. LIN (2012a). Combined processes of two-stage Fentonbiological anaerobic filter-biological aerated filter for advanced treatment of landfill leachate. Waste Manage., $32,2401-2405$.

WANG Y., X. LI, L. ZHEN, H. ZHANG, Y. ZHANG et C. WANG (2012b). Electro-Fenton treatment of concentrates generated in nanofiltration of biologically pretreated landfill leachate. J. Hazard. Mater., 229-230, $115-121$.

WEISS E. (2006). Étude cinétique de la dégradation électrochimique de composés organiques sur l'anode de diamant dopé au bore: Application à la dépollution d'effluents aqueux. Thèse de doctorat, Univ. Paul Sabatier, Toulouse III, France, 279 p. 
WREFORD K., J.W. ATWATER et L.M. LAVKULICH (2000). The effects of moisture inputs on landfill gas production and composition and leachate characteristics at the Vancouver Landfill Site at Burns Bog. Waste Manage. Res., 18, 386-392.

XIAO S., J. PENG, Y. SONG, D. ZHANG, R. LIU et P. ZENG (2013). Degradation of biologically treated landfill leachate by using electrochemical process combined with UV irradiation. Sep. Purif. Technol., 117, 24-29.

XIE B., B.Y. LV, C. HU, S.B. LIANG, Y. TANG et J. LU (2010a). Landfill leachate pollutant removal performance of a novel biofilter packed with mixture medium. Bioresour. Technol., 101, 7754-7760.

XIE B., Z. LV, B.Y. LV et Y.X. GU (2010b). Treatment of mature landfill leachate by biofilters and Fenton oxidation. Waste Manag., 30, 2108-2112.

YAMAZAKI I. et L.H. PIETTE (1991). EPR spin-trapping study on the oxidizing species formed in the reaction of the ferrous ion with hydrogen peroxide. J. Am. Chem. Soc., $113,7588-7593$.

YING W.-C., R.R. BONK, V.J. LLOYD et S.A. SOJKA (1986). Biological treatment of a landfill leachate in sequencing batch reactors. Environ. Prog., 5, 41-50.

YOON J., S. CHO, Y. CHO et S. KIM (1998). The characteristics of coagulation of Fenton reaction in the removal of landfill leachate organics. Water Sci. Technol., $38,209-214$.
ZAZOULI M.A., Z. YOUSEFI, A. ESLAMI et M.B. ARDEBILIAN (2012). Municipal solid waste landfill leachate treatment by fenton, photo-fenton and fenton-like processes: Effect of some variables. Iranian J. Environ. Health Sci. Eng., 9 (1), 3.

ZHANG D.-B., X.-G. WU, Y.-S. WANG et H. ZHANG (2013). Landfill leachate treatment using the sequencing batch biofilm reactor method integrated with the electroFenton process. Chem. Pap., 68, 782-787.

ZHANG H., D. ZHANG et J. ZHOU (2006). Removal of COD from landfill leachate by electro-Fenton method. J. Hazard. Mater., 135, 106-111.

ZHANG H., X. RAN, X. WU et D. ZHANG (2011). Evaluation of electro-oxidation of biologically treated landfill leachate using response surface methodology. J. Hazard. Mater., 188, 261-268.

ZHANG H., X. RAN et X. WU (2012a). Electro-Fenton treatment of mature landfill leachate in a continuous flow reactor. J. Hazard. Mater., 241-242, 259-266.

ZHANG H., X. WU et X. LI (2012b). Oxidation and coagulation removal of COD from landfill leachate by Fered-Fenton process. Chem. Eng. J., 210, 188-194.

ZHAO X., J. QU, H. LIU, C. WANG, S. XIAO, R. LIU, P. LIU, H. LAN et C. HU (2010). Photoelectrochemical treatment of landfill leachate in a continuous flow reactor. Bioresour. Technol., 101, 865-869. 